\title{
L1 EN EL AULA DE L2: ¿POR QUÉ NO?
}

\author{
$\mathrm{M}^{\mathrm{a}}$ Mar Galindo Merino \\ Universidad de Alicante \\ Grupo AcqUA \\ Mar.Galindo@ua.es
}

\begin{abstract}
Resumen
Una de las cuestiones más polémicas que ha recorrido la enseñanza de lenguas modernas a lo largo de todo el siglo XX ha sido, sin duda, el debate sobre el empleo de la primera lengua de los estudiantes en el aula de idiomas. A ese respecto, han sido muchos y muy variados los argumentos a favor y en contra. Nuestro artículo se propone revisar tanto las razones que se han argüido para rechazar la presencia de la Ll en la clase de lenguas extranjeras como las que se aducen para incluirla, con especial atención a las aportaciones más recientes de la Teoría sociocultural del aprendizaje de idiomas. No se trata de un tema meramente lingüístico, sino que entran en juego también factores de índole psicológica, social y cultural, que vinculan directamente este asunto con los fenómenos de multicompetencia y plurilingüismo.

PALABRAS CLAVE: lengua materna, primera lengua, cambio de código, alternancia lingüística, aula de segundas lenguas.
\end{abstract}

\begin{abstract}
One of the most controversial issues involving the teaching of modern languages along the $20^{\text {th }}$ century has been, doubtless, the debate on the use of the students' mother tongue in the language classroom. Indeed, there has been a wide variety of arguments for and against of first language use. Thus, our paper aims to review both perspectives, focusing especially on the recent contributions of the Sociocultural theory. We will cover not only the linguistic dimensions of this topic, but also the psychological, social and cultural elements that relate codeswitching in the language classroom with multicompetence and multilingualism.
\end{abstract}

KEY WORDS: mother tongue, first language, codeswitching, linguistic alternance, second language classroom.

\section{La lengua materna del alumno en el aula de idiomas}

La lengua materna de quienes aprenden una lengua extranjera es un elemento que no podemos desvincular de los procesos de aprendizaje. Desde las primeras noticias que tenemos de aprendizaje lingüístico en la historia de la Humanidad, que datan de hace más de tres mil años, la lengua materna de los aprendices ha estado presente, como ocurría en las listas de vocabulario bilingües confeccionadas para que los acadios aprendieran el idioma de los sumerios un milenio antes de nuestra era. Este factor ha sido tratado en mayor o 
menor medida, directa o indirectamente, en la formulación de los distintos enfoques y métodos de enseñanza de idiomas (Galindo Merino, 2009), así como en las teorías que intentan explicar la adquisición de segundas lenguas. Sin embargo, la historia del uso de la L1 en el aula de L2 ha estado marcada por su largo destierro que, prácticamente, recorre de principio a fin el siglo XX. El veto a la lengua de los estudiantes se ha manifestado en distintos grados (Cook, 2001a). El más radical, la absoluta prohibición de emplear la Ll en clase de L2 (Ban the L1 from the classroom), únicamente posible cuando el profesor no conoce la lengua de sus alumnos (en otras circunstancias, resulta poco probable que no se recurra a ella). En su versión más suave, esta consigna se nos presenta como un intento de máxima reducción del uso de Ll en el aula (minimise the L1 in the classroom), es decir, de limitar su uso al mínimo. Por último, una visión más optimista de este vacío a la lengua materna es la cláusula de potenciar al máximo la L2 en el aula, enfatizando las ventajas del rendimiento de la L2 en lugar de los peligros de la L1. En todas estas posturas se asume, explícita o implícitamente, que la lengua meta es positiva y, la lengua primera, negativa. De hecho, como apunta Elsa Auerbach en su famosísimo artículo de 1993, a pesar de la gran oposición actual al English-Only movement por parte de los defensores de la educación bilingüe y el apoyo a los derechos lingüísticos ("advocacy for language rights", Auerbach, 1993: 9), muchos profesores de inglés en Estados Unidos continúan pensando que el inglés "is the only acceptable medium of communication within the confines of the ESL classroom" (Auerbach, 1993: 9).

Como ha ocurrido en otras áreas de la historia del aprendizaje de idiomas, la consideración del papel de la primera lengua en el aula ha sufrido un movimiento pendular tanto en los métodos de enseñanza como en las teorías de aprendizaje (Duff, 2005) y no ha estado exento de polémica en todo este tiempo ("there have been cyclical fluctuations [...] often determined by political rather than pedagogical factors", Auerbach, 1993: 12; "...the place of the mother-tongue as been subject to violent swings of the pendulum", Prodromou, 2001; "There have always been contradicting views about whether to use the mother tongue of the students in the foreign language classroom", Tang, 2002: 1; "constant fluctuations", Rodríguez Juárez y Oxbrow, 2008). Esta observación conduce a Costas Gabrielatos (2001: 6) a catalogar el uso de la Ll como la manzana de la discordia, que ha sido el origen de arduas discusiones durante décadas.

A pesar de este vaivén, en la formación de muchísimos profesores de idiomas, especialmente relacionados con la enseñanza del inglés, pesa la tradicional consigna de no emplear la Ll en sus clases ("This advice is echoed in 
almost every teaching manual", dice Cook, 2001b: 4; "Extensive, if not exclusive, use of the target language is a long-standing tenet of second language (L2) teaching", Edstrom, 2006: 276), en lugar de plantearse cuándo y cómo usarla para lograr el máximo beneficio de los estudiantes (Larrea, 2002). "The matter is rather complex and the only thing I would prohibit in the present debate is the word "prohibition"', decía un profesor italiano en un foro de debate sobre el uso de la Ll, al que aludiremos en breve (Stanley, 2002). No son pocos los profesores que se han rebelado ante esta imposición, tachándola de absolutista, pues, ¿quién tiene el derecho de decidir qué lengua hablar? El hecho de servirse de la Ll en el aula no significa que se convierta en la lengua dominante, sino que su empleo es recomendable dentro de unos límites y para fines pedagógicos. El fracaso en el aprendizaje de idiomas se ha achacado muchas veces al abuso de la Ll y no a la falta de motivación de los alumnos para utilizar la L2, por ejemplo.

Sea como fuere, la prohibición de hablar la lengua materna ha sido a la vez un principio metodológico y una regla de comportamiento en clase por la que millones de aprendices han pagado otras tantas sumas de dinero. $\mathrm{Y}$ tanto aprendices como instructores se preguntan: ¿Dicha prohibición favorece el aprendizaje?

Independientemente del camino que recorran los teóricos y la prescripción de cada método de enseñanza, y con relación a dicha prohibición, lo cierto es que en cada rincón del mundo hay un profesor de idiomas (nativo y no nativo) con su propia y peculiar visión del papel de la lengua nativa de sus alumnos. Algunos de ellos, con declaraciones tan evidentes como la de Wolfgang Butzkamm en su artículo "How I changed my mind and started using the mother tongue in the foreign language classroom"1.

Otros, en foros de internet y blogs, dejan entrever su experiencia como profesores de lenguas aquí y allá, con políticas educativas que prohibían el uso de la Ll de los estudiantes, lo cual creaba situaciones de malestar entre los alumnos si se seguían fielmente las normas dictadas por las autoridades académicas, o entre el profesorado cuando tales pautas se infringían (Burden, 2000). El foro de discusión sobre el uso de la Ll "Using the First Language in Second Language Instruction: if, when, why and how much?", editado por Karen Stanley en 2002 para la revista electrónica TESL-EJ, Teaching English as a Second or Foreign Language, recibió más de treinta entradas de alumnos, profesores en formación y en activo, editores y lingüistas de uno y otro lado del Atlántico que entre mayo de 2000 y junio de 2001 discutieron los pros y los

1 Publicado en http://www.fremdsprachendidaktik.rwth-aachen.de/Ww/changed.html [22/02/2010]. 
contras de este uso. Como la misma Karen Stanley (2002) reconoce, hay un amplio abanico de opiniones respecto al grado de uso de la Ll en el aula: desde quienes defienden la prohibición total de emplear la primera lengua hasta el resto de propuestas que prefieren un uso limitado. Todo ello dependerá, según Stanley, de factores como las normas sociales y culturales, la motivación de los estudiantes y sus objetivos, la edad, la metodología empleada, el contexto de aprendizaje, el nivel y el perfil monolingüe o multilingüe del grupo. A estos factores añade Portaluri (en Stanley, 2002) la Ll de los aprendices, la ratio de estudiantes por profesor y la duración de la clase y del programa en que se incluye, entre otros.

El profesor Glenn Levine (2003) sugiere, además, el papel de la formación didáctica recibida, los conocimientos sobre la adquisición de segundas lenguas, la política del centro de estudios y la experiencia docente, aunque reconoce que muchas veces la práctica diaria responde más a las intuiciones del profesor en cada caso que a cualquier otro factor.

Y la verdad es que, a pesar de la extendidísima recomendación impuesta de no recurrir a la L1 en el aula de L2, la realidad es bien distinta ${ }^{2}$. Así lo reconoce Francine Chambers (1991: 27): "The belief that the foreign language should be used as the teaching and learning medium appears to be shared by many teachers and yet, despite this commonality of intent, practice varies greatly". Para los profesores de inglés como lengua extranjera resulta casi imposible no emplear la lengua nativa de los aprendices especialmente en los niveles más bajos (Tang, 2002: 1). Edgar Larrea (2002) presenta el panorama de la enseñanza de inglés como lengua extranjera en Latinoamérica, donde en la mayoría de ocasiones, alumnos y profesor comparten la primera lengua. Sin embargo, la dinámica de estas clases monolingües queda lejos de las aulas de inglés multilingües, que se dan tanto en Estados Unidos como en Europa, y en donde la política de EnglishOnly es perfectamente válida cuando el inglés no es solo lengua meta y de instrucción, sino que desempeña también el papel de lengua franca. Además, la cuestión de cuánta lengua meta emplea el profesor y en qué contextos lo hace, lo mismo que cuánta lengua meta esperan utilizar los aprendices, está indisolublemente unida a otras prácticas de clase (Levine, 2003: 343).

Lo cierto es que la enseñanza de lenguas modernas ha tomado como válidos todos los presupuestos asumidos por la enseñanza de inglés como segunda

2 Gill (2005: 2) recoge el testimonio de una colega eslovaca que observó una clase de francés en Londres en la que, exactamente, se emplearon únicamente siete palabras de la lengua meta durante toda la sesión. Considera que, a pesar de los tabúes, las normas, los sentimientos de culpa y las prohibiciones, en muchos contextos educativos la tónica es el uso predominante de la primera lengua. 
lengua, sin tener en consideración las circunstancias particulares de cada contexto y de cada grupo de alumnos, lo cual ha desembocado en la creación de mitos y ficciones monolingües, totalmente alejados de la situación real del aula (Belz, 2002: 211; Çelik, 2008: 75), en la que, muchas veces, el profesor comparte la lengua materna con el alumno. Baste como ejemplo el libro de Tony Lynch Communication in the Language Classroom (1996), que analiza la interacción en el aula (especialmente, la negociación) y los discursos de profesor y alumno. No cita ni una sola vez el uso de la lengua materna, cuando los docentes de grupos monolingües saben de sobra que la L1 forma parte del discurso habitual en la clase de lenguas extranjeras (Cole, 1998). Ello lleva a Miles (2004: 2) a distinguir, para la enseñanza de idiomas, entre un enfoque monolingüe (Monolingual Approach) caracterizado por el uso exclusivo de la lengua meta, y un enfoque bilingüe (Bilingual Approach), que incluye a los partidarios del empleo de la primera lengua en el aula.

Por ello, a pesar de las discrepancias existentes en torno al uso de la $\mathrm{Ll}$ en el aula de L2, "...the current trend suggests that it is necessary to at least reevaluate the role of the students' $\mathrm{Ll}$ in the process of foreign language learning" (Zacharias, 2003: 38), ya que en torno a esta cuestión las posturas adoptadas han tendido a los extremismos ("There seems to be an all-or-nothing view", Auerbach, 1993: 15; "...most teachers either fall on one side or the other", Cunningham, 2000; "...current tendencies to completely avoid L1 use in student interaction", Antón y DiCamilla, 1999: 245; "The L1 must be avoided at all costs", considera una de las participantes en el estudio de Macaro, 2001: 545 ; "...the risk is too great in some situations for the teacher to adhere strictly to a principle", Chambers, 1991: 29; "no one should go to extremes in the debate of the use of L1 or L2", Wang, 2002: 6; "FL is not an all-or-nothing affair", Butzkamm, 2003: 37). En consecuencia, analizaremos a continuación los argumentos en contra y a favor de dicho empleo, con objeto de arrojar algo de luz sobre este debate.

\section{Razones para rechazar la L1 en el aula de L2}

"The teacher stares at the wide eyes of his new students, "Class. We are here to learn English. As of today, you are not to use any Japanese in this room. This is an 'English-Only' class." And it is from that moment, I would argue, that the class is lost". Con este explícito principio da comienzo Robert Weschler (1997: 1) a su artículo sobre la enseñanza de inglés en Japón y la metodología empleada. Y esta situación que él tan claramente describe resulta más común de lo que imaginamos en la moderna glotodidáctica. 
La asunción de que la lengua materna influye sobre la L2, y que esa influencia es siempre negativa, es una de las creencias más extendidas sobre el aprendizaje de idiomas, junto con la idea de que cuanto mejor es un estudiante, menos necesita el apoyo de su lengua materna, hasta tal punto que podemos decir que uno de los signos de la enseñanza de idiomas del siglo XX es su postura anti-L1, como apunta Cook: "This anti-Ll attitude was clearly a mainstream element in twentieth century language teaching methodology" (Cook, 2001a: 2).

Uno de los argumentos más ampliamente utilizados para evitar el uso de la lengua materna de los aprendices es que ello podría producir dependencia por parte del alumno, de modo que dejaría de intentar comprender el input de L2 (Auerbach, 1993: 20; Çelik, 2008: 76; Rolin-Ianziti y Varshney, 2008: 262). Esta idea es la que recoge Anthea Tillyer en el foro de Stanley (2002). Para ella, los aprendices han de estar preparados para afrontar momentos incómodos en el aula al no entender la lengua meta, pero "también la vida está llena de estrés y dificultades". Si el profesor habla la L1 para que el alumno se sienta más tranquilo, en realidad está equivocado, pues estará alargando el período de tiempo en que el aprendiz quedará en el miserable limbo de ser incapaz de comunicarse en L2. Así lo expresa Dick Tibbetts, de la Universidad de Macao (en Stanley, 2002): "If the Ll is the language of action and power, the language of classroom organisation and discipline and the language through which the L2 is mediated, then it is rather a bad example to students and gives the message that the L2 is merely a classroom subject, not a real useful language".

Elsa Auerbach, una de las autoras que con más profusión ha tratado el tema, considera que la cuestión de la(s) lengua(s) hablada(s) en clase ha de ser negociada con los aprendices, puesto que el uso de la Ll puede crear tensión, transmitir la sensación de ralentización del aprendizaje y de pérdida de tiempo y desencadenar, en última instancia, actitudes negativas por parte de los alumnos, especialmente en los niveles avanzados (1993: 23). Muy similares son los argumentos esgrimidos por Sheona Hopkins (1988: 19) para rechazar el empleo de la L1. Para Charlene Polio (1994: 155) constituye un paso atrás emplear en el aula cualquier lengua que no sea la lengua objeto de aprendizaje. Ello equivale a transmitir a los alumnos la idea de que la lengua meta no es un vehículo real de comunicación como sí lo es la primera lengua (Franklin, 1990: 20), además de privarles de un valioso input en lengua meta y de las correspondientes oportunidades para procesarlo y crear situaciones de comunicación en la L2 (Kim y Elder, 2005: 356; Çelik, 2008: 75; Rolin-Ianziti y Varshney, 2008: 260).

Bajo estos presupuestos, como decimos, de manos de la investigación en Adquisición de segundas lenguas la Ll de los aprendices de lenguas extranjeras sufrió un injusto destierro del aula en el último tercio del siglo pasado, con el 
caso paradigmático de la enseñanza de inglés (bajo los postulados del famoso English-Only movement, Auerbach, 1993). En concreto, se descartaba el uso de la Ll para cualquier actividad en la que interviniera (incluso, de tipo contrastivo): las explicaciones gramaticales, la comparación interlingüística, los ejercicios de traducción en cualquiera de sus modalidades o los glosarios bilingües estaban excluidos, por principio, del aula, si bien la práctica docente realizada por profesores en todo el mundo con frecuencia contravenía tal ortodoxia (Martín Martín, 2004: 16). Como principales razones para tal ausencia se esgrimen tres argumentos:

- La vinculación del uso de la Ll con métodos caídos en descrédito como el de gramática-traducción, en los que el componente oral no se desarrollaba.

- La influyente aportación de Krashen y sus colegas, cuya investigación ponía de manifiesto la total irrelevancia de la primera lengua en el aprendizaje de una segunda, creando un sentimiento de culpabilidad entre los profesores que recurrían a la Ll como instrumento de comunicación y aprendizaje en el aula.

- La ingente campaña de defensa del uso exclusivo de la L2, y que primordialmente partía de las principales editoriales del mundo de habla inglesa a lo largo de los años setenta y ochenta, empeñadas en favorecer claramente sus intereses comerciales dadas las ventajas económicas de prescindir de la Ll a la hora de confeccionar los materiales. La innegable calidad de muchos de estos manuales se veía mermada por las deficiencias derivadas de la desatención a la realidad lingüística del aprendiz, que, en la mayoría de casos, había de ser subsanada por el profesor impartiendo gran parte de la clase en Ll y produciendo, en consecuencia, el efecto inverso.

Por ello podemos decir que, muchas veces, el destierro de la Ll obedece a razones comerciales: las editoriales crean libros de texto monolingües en consonancia con teorías lingüísticas que favorecen este planteamiento, puesto que resulta más barato la producción de material docente con vocación de distribución global. Este factor ha sido puesto de manifiesto por numerosos investigadores (Weschler, 1997; Martín Martín, 2001):

... it is the Dulay, Burt and Krashen position that has won the day. Proof of this statement can be seen by examining textbooks for foreign and second language teaching available today from publishers. They are for the most part, solely in English, making no reference to the $\mathrm{Ll}$ of the learners. This practice, of course, suits publishers because it enables them to sell the same textbooks all over the world, thus increasing their profits [...] Publishing solely monolingual texts without contrastive information and arguing that they are the best choice for all situations is, therefore, of doubtful legitimacy. (Sheen, 2000: 103). 
Razones económicas y profesionales son las que aduce Merton L. Bland (en Stanley, 2002) para no hablar la L1 en clase de L2: al profesor se le paga para enseñar la L2 (inglés, en su caso), no la L1. Cuando la clase constituye una inmersión real en la lengua meta, el alumno considera la L2 como un verdadero sistema de comunicación, más que como un objeto de estudio. Más adelante, otro profesor de idiomas, Jeremy Taylor, comenta que los alumnos que han estudiado idiomas con profesores que no hablaban su L1 demuestran mejores resultados en el dominio de la L2, porque toda la comunicación entre docente y discente ha de llevarse a cabo en la lengua meta.

Atendiendo a los factores económicos que explican este rechazo a la L2, Weschler (1997: 6) también señala razones de esta índole: "From the institution's point of view, the native speakers are being paid good money to supply the students with optimal exposure to natural English, something supposedly only a native speaker can do [...]; the institution wants to get its money's worth".

Para Larrea (2002), en cambio, el rechazo de la Ll procede de la idea de que su uso impide la adquisición, junto con la creencia de que la transferencia de la lengua materna es la causa de los errores de los alumnos: "There was little (if any) we could get from using $\mathrm{Ll}$ in the communicative language class" (Larrea, 2002: 2). Él constata que, a pesar de la prescripción, los alumnos traducen a su lengua materna, y la emplean como estrategia en el aprendizaje de una lengua extranjera.

En Teaching monolingual classes (1993a: 12-13), Atkinson muestra una serie de razones para llevar a cabo la clase de L2 en L2, que básicamente se resumen en que la exposición a la L2 promueve el aprendizaje, y el empleo de la Ll en el aula limita la cantidad de lengua meta disponible para el alumno: "If English is not the main language used in the classroom, the learners are not going to learn very much English". Por ello, "it's better to use English supported by one of these techniques [gesture, mime] than to go into the L1" (Atkinson, 1993a: 103). Esta enérgica defensa del uso de la L2 está relacionada con la necesidad de proporcionar a los aprendices el máximo input posible en lengua meta no solo durante las explicaciones de clase y la realización de actividades, sino también para el manejo del aula y el mantenimiento de la disciplina (Ellis, 1984: 133; Chaudron, 1988: 121; Wang, 2002). El cambio a la L1, incluso en funciones lingüísticas secundarias, supone privar a los aprendices de un valuable input en L2 (Ellis, 1984; Kim y Elder, 2005; Rolin-Ianziti y Varshney, 2008).

Turnbull y Arnett (2002: 205) citan una larga serie de estudios que muestran una correlación directa entre el uso de la lengua meta por parte del profesor y la adquisición de la L2, lo cual constituye sin duda el argumento 
fundamental a la hora de defender el mayor uso posible de la segunda lengua en el aula. De hecho, algunos profesores en prácticas entrevistados por Turnbull coincidían en que "...immersing oneself in the TL is the most effective way to learn a language" (Turnbull y Arnett, 2002: 210). Por ello, resultan chocantes los resultados de algunos estudios que muestran que el uso de la primera lengua puede favorecer la adquisición de una segunda lengua, aunque para Swain y Lapkin (2000) esta situación es paradójica solo en apariencia, y su artículo en Language Teaching Research trata de explicar esta contradicción.

Aparte de las razones aducidas por los expertos, también los docentes consideran una serie de factores para restringir al máximo el uso de la lengua materna en el aula. La investigación de James Corcoran en academias de inglés en Brasil, en las que entrevistó a veinticinco profesores, pone de manifiesto algunos de ellos (Corcoran, 2009: 11):

(a) It limits opportunities for student exposure to the TL.

(b) The importance of students' learning negotiation strategies in the $\mathrm{TL}^{3}$.

(c) Student demand for TL use.

(d) Avoidance of 'opening the floodgates' to rampant Ll use in class.

(e) Prior teacher TL learning experiences.

(f) Learners' TL proficiency level.

(g) Discouragement of translation.

(h) Avoidance of focus on grammar.

(i) Teacher TL proficiency.

(j) Teacher education.

(k) Institutional policy.

Nosotros añadiríamos también Teacher MT proficiency, es decir, los conocimientos de la lengua materna de los alumnos que tenga el profesor.

3 La negociación del significado es uno de los factores con los que González García (2009: 10 y ss.) vincula el empleo de la L2: el uso de la primera lengua hace que los alumnos no sientan la necesidad de negociar el significado en la lengua que están aprendiendo, impidiendo, en consecuencia, la adquisición. Sin embargo, también contempla la posibilidad de que la negociación esté guiada por la primera lengua, lo cual restaría fuerza a la hipótesis anterior. De hecho, su conclusión va más en esta línea:

El aspecto que claramente consideramos inapropiado e injustificado es la marginación de la Ll en las clases de ELE con el pretexto de que, al recurrir a la Ll, los aprendices evitan la negociación. [...] Una actitud más permisiva hacia el uso de la Ll es igualmente efectiva a la hora de fomentar el reconocimiento de nuevas palabras y su adquisición, siempre y cuando el proceso de negociación guiada, con un enfoque secundario en la forma, sea aplicado con criterio. La prohibición de la Ll eliminaría la utilidad de la negociación guiada, reforzada con el uso esporádico y limitado de la Ll. 
Robert Phillipson explica el rechazo de la primera lengua por razones políticas: la consolidación del control neocolonial británico tenía como componente clave el dominio del inglés (siendo "inglés" sintácticamente el sujeto de dominio y no el objeto dominado). Cita directamente un congreso celebrado en Uganda en 1961 del que, según él, emergieron cinco principios clave que subyacen a la enseñanza de inglés actual (Phillipson, 1992: 185):

- English is best taught monolingually.

- The ideal teacher of English is a native speaker.

- The earlier English is taught, the better the results.

- The more English is taught, the better the results.

- If other languages are used too much, standards of English will drop.

A ojos de Phillipson, estos principios se han convertido en la piedra angular de la enseñanza de inglés en todo el mundo y explican en buena parte el rechazo a emplear otras lenguas en el aula de idiomas.

Charlene Polio (1994) le da la vuelta a este argumento en su respuesta al artículo de Elsa Auerbach, aduciendo que el uso de otras lenguas en el aula es también un modo de perpetuar relaciones de poder: el no emplear la lengua meta es una manera de transmitir determinados mensajes al alumno ("esta lengua es difícil"; "nunca la aprenderás"; "no vale la pena que te esfuerces en aprender este idioma", en Polio, 1994: 155). Auerbach, en su respuesta final al comentario de Polio, discrepa.

Según Julie Belz (2002), el uso de la Ll y de otras lenguas en el aula se ha asociado siempre con deficiencias en el aprendizaje de lenguas (Cook, 1999), basándose en una concepción deficiente del estudiante de idiomas, en constante comparación con el hablante nativo, modelo homogéneo e ideal al que debe tender ("...foreign language teachers explicitly link L1 use to the allegedly deficient nature of the learner", Belz, 2002: 216). Ello ha dado lugar a abundantes caracterizaciones del aprendiz de lenguas que remarcaban sus fallos y problemas. Es decir, el estudiante de idiomas se ha visto a menudo definido por el incompleto éxito que alcanza durante el proceso de aprendizaje, al final del cual, normalmente no logra la proficiencia total en la L2. Esta concepción hace que los cambios de código L1/L2 del alumno de idiomas no se achaquen al uso de estrategias discursivas conscientes, como ocurre entre hablantes bilingües, sino a su déficit y falta de competencia comunicativa. Más adelante comentaremos algunos de estos aspectos al mencionar el concepto de multicompetencia y la nueva visión que trae tanto del aprendiz como del aprendizaje de lenguas. Belz (2002: 217) describe al aprendiz multicompetente así: "I paint a picture of the language learner as a multicompetent language 
user who carefully and consciously uses multiple linguistic codes not necessarily for reasons of deficiency and failure, but rather to play, represent, experiment, create, juxtapose, learn, and grow".

Muchas de las razones para evitar el uso de la Ll en el aula son aplicables siempre que el profesor de idiomas conozca la lengua materna de sus alumnos, circunstancia difícilmente factible en los casos de alumnado multilingüe o de profesor nativo que enseña su idioma en distintos países (Cook, 2001b). Puesto que el conocimiento de la $\mathrm{Ll}$ de los aprendices no es un requisito para enseñar una lengua, muchos métodos, enfoques, teorías y materiales no tienen en cuenta este factor. Además, en aulas plurilingües, los conocimientos lingüísticos del profesor suelen limitarse, con suerte, a unas pocas lenguas, pero casi nunca abarcan todos los idiomas presentes en el aula, por lo que el uso de alguna L1 implicaría automáticamente excluir a otros de esa posibilidad.

Según Atkinson (1995: 2), los más entusiastas defensores de las políticas docentes English-Only son aquellos que desconocen la realidad del aula monolingüe de idiomas, y afirma que cualquiera que enseñe en niveles bajos a un grupo que comparta la $\mathrm{Ll}$ tiene verdaderas dificultades para convencerse a sí mismo de que el uso exclusivo de la lengua meta es el medio más eficaz de conseguir el aprendizaje. Del mismo modo, Cook entiende que un argumento de peso para desterrar la Ll del aula es la presencia de alumnado multilingüe, pero "The practical reasons for avoiding the first language in a multilingual class do not justify its avoidance in classes with a single first language. It is hard to find explicit reasons being given for avoiding the first language in these circumstances" (Cook, 2001b: 154).

En este sentido, resulta conveniente hacer referencia a la importancia del contexto. Dick Tibbetts, profesor de inglés en China (en Stanley, 2002), establece una diferencia contextual en el uso de la Ll. Si bien le parece tolerable en contextos de segunda lengua, para suavizar el choque cultural al que se ve sometido el alumno, no lo considera aceptable en contextos de lengua extranjera, en los que la clase es la única fuente de input para los alumnos, y hay que mostrarles que el inglés es una verdadera lengua de comunicación. De hecho, su experiencia en la Universidad de Macao le dice que los alumnos que habían aprendido inglés con profesores que no hacían empleo de la Ll generalmente entraban en las clases de nivel más alto, mientras que aquellos estudiantes que habían adquirido el inglés con mediación del chino solían quedarse en las clases de nivel intermedio.

Un caso curioso es el que relata Cindy LaVan (2001) acerca de los programas de inmersión en español en Minnesota. Según ha podido constatar por su experiencia como profesora junto con los testimonios de sus colegas, conforme 
aumenta la competencia lingüística de los aprendices en español (L2) se aprecia un aumento de la Ll (inglés) en el aula, que ella achaca a la falta de motivación, lo cual da lugar a un círculo vicioso: más Ll, menos motivación; menos motivación, más Ll. Si bien en los niveles más bajos de estos programas resulta relativamente fácil hacer que los alumnos empleen masivamente la L2, la voluntad de socializar con los compañeros y la menor atracción hacia la lengua meta hace que la Ll surja con más facilidad, sobre todo en forma de cambios de código, y ejerza una influencia negativa en la producción en L2 de los aprendices. Esta disminución en el uso de la L2 en los niveles más altos de los programas de inmersión ha sido apuntada también por expertos como Merrill Swain (1985) y Elaine Tarone (Tarone y Swain, 1995), que ofrecen una explicación sociolingüística de este fenómeno. Según ellas, las relaciones L1/L2 en situaciones de inmersión dan lugar a una comunidad de habla regida por una clara situación de diglosia, en la que la L2 desempeña el rol de lengua formal para fines académicos, mientras que la Ll es una suerte de vernáculo a través del cual los aprendices establecen su identidad y se integran en el grupo. Quienes no emplean la Ll se desmarcan de ese grupo automáticamente, por lo que son rechazados. Ello explica también la aparición de artículos como el de Jennifer Straub (2006), centrado en aumentar la motivación de los aprendices para hablar francés en contexto de inmersión.

Ante tal situación, LaVan (2001: 3) se pregunta qué ocurriría si el papel de idioma vernáculo lo desempeñara otra variedad de la L2, y no exclusivamente la Ll, puesto que en otros programas de inmersión se daba el caso de que los alumnos socializaban en inglés vernáculo, y reservaban un inglés más formal para el aula. El problema, en consonancia con lo apuntado por Tarone y Swain (1995), es que los alumnos no tenían acceso a ese vernáculo en el aula ni fuera de ella, por lo que el único recurso posible era la Ll. Por ello, ofrece una serie de estrategias para el profesor dirigidas a aumentar el uso de la L2 en el aula, que son las siguientes, según las enumera ella misma:

1. Create a classroom and school context with clear expectations for L2 use.

2. Establish clear separation of the two languages in the classroom.

3. Acknowledge that neither rewards nor punishments affect behavior positively.

4. Set language learning objectives.

5. Develop non-academic vocabulary.

6. Organize classroom activities and provide opportunities that maximize students' L2 output.

La insistencia en emplear al máximo la lengua meta se debe, en parte, a que en muchos contextos el abuso de la L1 (unbridled use of the L1 dice Macaro, 
2001: 532) es todavía una de las grandes barreras que han de superarse en el aula de idiomas (Atkinson, 1993a; Cole, 1998; Larrea, 2002; Turnbull y Arnett, 2002; Nation, 2003; Rolin-Ianziti y Varshney, 2008; Çelik, 2008; Carless, 2008). A este respecto, Butzkamm (2003: 32) saca a colación el refrán inglés Give the devil an inch and he'll take a mile, equivalente a nuestro coloquial "das la mano y te cogen el brazo", como explicación metafórica del abuso de la primera lengua que puede producirse en el aula de L2. Sandra Fotos, editora de un estudio sobre bilingüismo en Japón (2001), comenta la situación de la enseñanza de inglés en el país nipón: "... many teachers of English use considerably more Japanese than the target language during instruction [...] it might appear that the use of the $\mathrm{L} 1$ is exactly what is not needed to promote second language acquisition..." (Fotos, 2001: 329). Este uso parece estar más extendido en los países orientales, puesto que es en este contexto donde hallamos más trabajos acerca del uso de la primera lengua en la enseñanza comunicativa de inglés como lengua extranjera. Ello apunta hacia una dirección que apenas es mencionada en los textos sobre este tema: la influencia de la distancia lingüística L1/L2 en el empleo de una u otra lengua en el aula. Catherine Wang (2002) muestra en su ensayo sobre la enseñanza de inglés en Taiwán el abuso de la primera lengua que tiene lugar en el aula, pues resulta más cómodo tanto al profesor como a los alumnos enseñar y aprender la lengua meta a través de la Ll. Ello tiene como consecuencia el empleo de una metodología más cercana a la técnica de gramática-traducción que a una verdadera enseñanza comunicativa de lenguas extranjeras. Quien sí apunta al factor de la distancia interlingüística como una razón fundamental de empleo de la lengua materna es Peter Dash (2002) en su trabajo sobre la enseñanza de EFL en Corea ("...the differences between English and Korean, linguistically and culturally are so great at times that it is not possible to explain every grammar point or cultural difference in English...").

Otros investigadores como Cain, Briane y Morgan (1998, citados en Macaro, 2001: 532) se preguntan cómo controlar el uso de la primera lengua una vez que se autoriza su empleo en el aula. El libro de Atkinson (1993a) sobre grupos monolingües ofrece soluciones para combatir el abuso de la Ll en situaciones concretas de clase, como también el artículo de Nation (2003), que posee un apartado específico llamado "Encouraging L2 use" donde sugiere distintas técnicas para superar los obstáculos que impiden que la clase se desarrolle en lengua meta.

A pesar del mencionado abuso, algunos autores mantienen que no hay ninguna razón por la que la primera lengua no desempeñe un determinado papel en el aula. El problema, como muchos apuntan (Atkinson, 1995: 2), es cuál es ese papel. Por ello es necesario, en palabras de Macaro (2001: 545), "a 
framework that identifies when reference to the $\mathrm{Ll}$ can be a valuable tool and when it is simply used as an easy option". Es la misma idea con la que concluye su artículo Robert Weschler (1997: 12): “...like any tool, it can be used skillfully or misused. It can have good or bad effects". Y es que la confianza excesiva en la primera lengua es uno de los peligros que acecha al aprendiz de lenguas, especialmente en la comisión de errores, según Costas Gabrielatos (2001: 8), por lo que hace una llamada a la cautela en su empleo:

Learners tend to rely on their existing language knowledge ( $\mathrm{Ll}$ and any other languages they can use) to help them understand the logic and organisation principles behind the target language. Uncritical use of $\mathrm{Ll}$ in the classroom (translation in particular) will reinforce this tendency. Ll use in the classroom needs to be handled with care exactly because it exerts a powerful influence on the learning process, as it seems that learners tend to treat it as the obvious starting point when learning a new language, and is a popular communication strategy (Ellis, 1985: 180-189). It is not a coincidence that a large number of the mistakes made by non-native users of English [...] tend to be the result of word-for-word translation, and transfer of cultural norms and communication conventions.

Además, si todas las funciones se realizan en la primera lengua, "there is little left to do in the L2" (Polio, 1994: 154).

También es posible, como añade Lorna Joy Swain (en Stanley, 2002), que los alumnos no empleen mucho la L2 en el aula porque no sepan, por lo que ofrece algunos ejercicios dirigidos a paliar tal carencia. Esa misma tendencia siguen autores como Francine Chambers (1991), Sagrario Salaberri (1993) o Paul Nation (2003), quienes proponen técnicas para introducir, desde el primer día, la L2 en clase. En su libro Uso del inglés en el aula, Salaberri propone a los profesores reflexionar sobre el uso que hacen de la L2 (en este caso, el inglés) en el aula, y les sugiere una serie de pautas para que la L2 sea empleada desde el primer día de clase, a fin de que los alumnos actúen de forma natural respecto a la lengua, se acostumbren a utilizar estrategias de comprensión y expresión y reconozcan el valor comunicativo que realmente tiene la lengua meta. Incorpora, además, unas fichas de seguimiento de actuación del profesor y autoanálisis del discurso, e incluye útiles listados con las expresiones más frecuentes utilizadas en el aula, para que el docente las introduzca en su práctica pedagógica en L2 desde el principio. Cabe la posibilidad, además, de que sean los propios alumnos quienes, por sus creencias, pidan una política de L2Only en el aula (Anthony, en Stanley, 2002).

En palabras de Luci Nussbaum (1991), el uso de la L1 en el aula contradice de algún modo las teorías sobre la adquisición de lenguas según las cuales el input abundante y el uso significativo de la lengua constituyen los procedi- 
mientos esenciales para su apropiación. Especialmente en niveles de aprendices principiantes en la enseñanza obligatoria, la lengua materna se emplea como vehículo de regulación de la enseñanza-aprendizaje y de las relaciones afectivas, ocupando así un espacio cualitativo (y, a veces, cuantitativo) superior al de la lengua meta. De hecho, muchos docentes insisten en que una de las claves es acostumbrar al alumno a entablar la interacción en el aula en lengua meta desde el primer día. El hecho de que los aprendices se habitúen a emplear la segunda lengua con el profesor hace que les resulte más natural su uso, de modo que surja casi espontáneamente como lengua preferente de comunicación. Esta realidad ha sido estudiada por la Sociolingüística en los contextos bilingües, y específicamente, en las interacciones entre hablantes que comparten las dos lenguas. Se ha constatado que los hablantes tienden a entablar relaciones en una u otra lengua, de modo que la interacción resulta más fluida con quien sabes que te hablará en una lengua que con quien cambia de idioma. En el marco del aula, ello se traduce en que los profesores que aplican políticas de uso exclusivo de la lengua meta hacen que se convierta en algo natural la conversación en L2, porque los alumnos saben que ése es siempre el vehículo de comunicación, de modo que no se plantean el empleo de la lengua materna.

En relación con este uso exclusivo de la lengua meta, la otra cara de la moneda apunta, como señalan algunos (Butzkamm, 2003; Wajnryb, 2004; Hitotuzi, 2006), a que las políticas educativas más restrictivas respecto al uso de la $\mathrm{L} 1$ procedan de un intento por acomodar al profesor nativo de L2 en un entorno en el que no está realmente preparado para lidiar con la primera lengua de sus alumnos: "One reason for the lack of reliance on the L1 has undoubtedly been convenience for the teacher" (Cook, 1999: 201). Esta tendencia puede asociarse a la enseñanza de inglés como lengua extranjera, situación en la que muchos docentes nativos de inglés se enfrentaban a clases en las que desconocían la lengua de sus alumnos, lo cual, lejos de sugerirles replantear su formación en idiomas, daba pie a la consolidación de la política English-Only y contribuía a potenciar su dominio político (Cook, 2001a; Miles, 2004). El triunfo de esta corriente metodológica lo achaca también Weschler (1997: 5) a la mayoría de profesores nativos de L2 sin conocimientos de la lengua del alumnado, y en ello coincide Simon Cole (1998): "the current 'use only L2' trend may have more to do with commercial expediency and low-level Ll competence among native-English speaking teachers than ideal pedagogy".

Es por ello por lo que muchos autores vinculan el rechazo hacia la lengua de los alumnos con el English-Only movement que con tanta fuerza se impuso en la enseñanza de inglés por parte de Estados Unidos y Gran Bretaña hace unas décadas. Autores como Swan (1985, citado en Barker, 2003) han señalado 
lo fácil que resultaba a los profesores nativos de inglés el enseñar su lengua por todo el mundo "sin la desagradable necesidad de tener que aprender otros idiomas", al amparo de este método. Hoy en día, la situación es distinta: independientemente del uso que se haga de la primera lengua, actualmente nadie duda del valor de conocer la lengua que hablan los alumnos, hasta el punto de que Cook afirma que "an L2 teacher who cannot use a second language may not be the best role model for the students" (2001b: 154).

Cook (200lb: 153) entiende que la clase de idiomas ha de ser un modelo de uso comunicativo de la lengua, desarrollando todas las funciones posibles en lengua meta para proporcionar a los aprendices el máximo input posible al tiempo que se transmite la sensación de que la L2 es realmente un vehículo de comunicación. Auerbach (1993: 14-15) lo resume muy bien: "The more students are exposed to English, the more quickly they will learn; as they hear and use English, they will internalize it and begin to think in English; the only way they will learn it is if they are forced to use it". Sin embargo, no se puede tratar a los alumnos como si fueran hablantes nativos, sino usuarios de la L2. La explicación que Cook (2001a) ofrece para explicar el destierro de la Ll en al aula de L2 se basa en tres teorías:

- La teoría de la adquisición de lenguas maternas (the L1 acquisition argument): el aprendizaje de una L2 ha de seguir el modelo de la adquisición de la L1, en la que no media ningún sistema lingüístico. Esta analogía justifica el destierro de la L1 en el aula de L2. Weschler (1997: 3) vincula esta idea con el trabajo de F. Gouin (1831-1896), precursor del método de respuesta física total de Asher, pero asume que no es válida en el caso de aprendices adultos.

- La teoría de la compartimentación lingüística (the language compartmentalization argument): un aprendizaje exitoso de segundas lenguas implica que el aprendiz separe totalmente L1 y L2.

- La teoría del uso comunicativo de la lengua meta (the second language use argument -the comunicative approach): los estudiantes no considerarán la L2 como un medio de comunicación viable y efectivo si el profesor no promueve su uso como tal. Por tanto, la máxima exposición a la lengua meta ha de ser el objetivo perseguido en la clase de idiomas, lo cual implica, automáticamente, desechar la comunicación en lengua materna ${ }^{4}$.

Estos argumentos pueden relacionarse claramente con las ideas de Stephen Krashen $(1980,1981,1985)$. Su Teoría de la construcción creativa o teoría del

4 En el ámbito de la enseñanza de inglés en China, Jinlan Tang (2002: 2) hablaba de esta idea como de: “...common criticism that using Chinese reduces the students' exposure to English". 
monitor se basa en cinco hipótesis bien conocidas, siendo la principal de ellas la hipótesis del input, directamente relacionada con la necesidad de prescindir en el aula de la Ll: se adquiere una lengua a base de oírla (hipótesis del input, fundamentada en el innatismo chomskiano), por lo que cualquier actividad que incluya el uso directo de Ll debe ser desechada; del mismo modo, las explicaciones gramaticales y el conocimiento explícito de la lengua solo ayudan a la adquisición en la medida en que, para su transmisión, se utilice la lengua objeto de estudio. Su postura es, pues, extrema respecto al papel de la Ll en el aprendizaje de una L2, y difícilmente sostenible a la luz de las investigaciones posteriores, que no dejan lugar a dudas de la influencia de la primera lengua tanto en los distintos niveles de la lengua (Galindo Merino, 2005a y 2005b) como en los efectos concretos que produce (comisión de errores, facilitación, evitación y abuso). Es más: tal como reconoce Rod Ellis, ninguna teoría de ASL puede ser considerada completa si no presta atención al conocimiento lingüístico previo. Y este conocimiento previo descansa sobre la sólida base de la lengua nativa del aprendiz.

\section{Razones para emplear la primera lengua en el aula de idiomas}

Frente a la solidez de muchos de los argumentos que acabamos de presentar, también son muchos los investigadores que reconocen que la lengua materna tiene su papel en la clase de idiomas; entre ellos, el mencionado Weschler. Es la impresión que lleva a David Barker (2003) a afirmar que "common sense dictates that $\mathrm{Ll}$ will always have a role to play in the language classroom". Aceptando ese papel, entre los autores que defienden los beneficios para el aula de idiomas de un uso moderado de la Ll de los estudiantes se encuentran Rivers (1981), Rod Ellis (1985), Rutherford (1987), Sheona Hopkins (1988), David Atkinson (1993a), Elsa Auerbach (1993), Véronique Castellotti (1997a), Simon Cole (1998), Marta Antón y Frederick DiCamilla (1999), William Schweers (1999), María De Guerrero y Olga Villamil (2000), José Miguel Martín Martín (2000a, 2000b), Robert Buckmaster (2000), Merrill Swain y Sharon Lapkin (2000), Costas Gabrielatos (2001), Ernesto Macaro (2001), Vivian Cook (2001a), Luke Prodromou (2001), Glenn Levine (2003), Mario Rinvolucri (2003), Paul Nation (2003), Nugrahenny Zacharias (2003), Storch y Wigglesworth (2003), Wolfgang Butzkamm (2003), Centeno-Cortés y Jiménez Jiménez (2004), Vincent Ferrer (2004), Richard Miles (2004), Thoms, Liao y Szustak (2005), Anne Edstrom (2006), Li-Ling Chen (2006), Nilton Hitotuzi (2006), Carless (2008), Çelik (2008), Rolin-Ianziti y Varshney (2008), Corcoran (2009) y Turnbull y Dailey-O'Cain (2009). Todos son o han sido profesores de idiomas. Tal como apunta Elsa Auerbach (1993: 23), muchos de ellos 
proceden de fuera de Estados Unidos, es decir, de países como Canadá o Inglaterra, donde existe una mayor defensa del multiculturalismo y la diversidad, lo cual se aplica también a la clase de lengua extranjera (especialmente, de inglés).

En este sentido, marcó un hito en la bibliografía especializada el mencionado artículo de Elsa Auerbach "Reexamining English Only in the ESL Classroom" (1993). Auerbach abogaba firmemente por el empleo de la Ll en la clase de inglés como segunda lengua, resaltando sus ventajas y equiparando el estatus de la L1 al de la L2. Ello crearía una atmósfera más humanística en el aula, que incluiría las condiciones afectivas y cognitivas que favorecen la adquisición. Del mismo modo, contribuiría a mantener la lengua nativa del aprendiz en el marco de un país anglohablante. Considera que el aprendizaje de una lengua ha de ser una suerte de transición natural de la L1 a la L2, y recomienda su uso especialmente en la enseñanza de segundas lenguas a adultos con poca o ninguna alfabetización, apoyándose en la investigación que confirma los beneficios del uso de la $\mathrm{Ll}$ en estos contextos ${ }^{5}$. Su posición se resume en el siguiente párrafo:

These findings concerning use of the $\mathrm{L} 1$ are congruent with current theories of second language acquisition. They show that its use reduces anxiety and enhances the affective environment for learning, takes into account sociocultural factors, facilitates incorporation of learners' life experiences, and allows for learner-centered curriculum development. Most importantly, it allows for language to be used as a meaning-making tool and for language learning to become a means of communicating ideas rather than an end in itself. (Auerbach, 1993: 20).

El texto de Auerbach ${ }^{6}$ supuso el pistoletazo de salida de una serie de estudios y artículos posteriores que ensalzaban las bondades del uso de la primera lengua en el aula como instrumento de mediación en la adquisición de una segunda lengua, especialmente en la enseñanza de ESL. A partir de entonces, "there appears to be an increasing conviction that the first language (L1) has

5 Esta situación se confirma en la práctica real de la enseñanza a inmigrantes no alfabetizados, a juzgar por los testimonios de numerosos docentes tanto en centros de adultos como de enseñanza secundaria, que manifiestan necesitar la lengua materna del alumno para facilitar los primeros estadios de aprendizaje.

6 El artículo de Auerbach recibió respuesta poco después por parte de Charlene Polio (1994). Polio considera que los postulados de Auerbach no son extrapolables a todos los contextos (por ejemplo, de lengua extranjera), y cuestiona conceptos como el de "uso", "permitido" e incluso el mismo "ESL". Cree que los usos para la Ll propuestos por Auerbach dejan poco margen a la lengua meta en el aula, y aboga por formar a los docentes de lenguas en técnicas de comunicación eficaz en L2, en consonancia con las modernas teorías de adquisición de segundas lenguas que ponen de manifiesto el impacto de la negociación del significado y de la modificación del input para el aprendizaje de idiomas. 
a necessary and facilitating role in the second and foreign language (L2) classroom", dice Schweers (1999: 1), quien vincula su aceptación de este recurso tanto a estos posteriores trabajos, citando expresamente el de Auerbach, como a su propia experiencia personal.

En general, casi todos los autores adoptan un enfoque más cognitivo que social a la hora de reivindicar este uso, aduciendo ejemplos relativos a procesos mentales que operan en el aprendiz durante la adquisición de una lengua no materna, aunque la tendencia se está invirtiendo, de modo que los trabajos más recientes incorporan el punto de vista social.

Uno de los autores más citados al hablar de estas cuestiones es David Atkinson (1987, 1993a), gran defensor del empleo de la Ll en la clase de idiomas. Atkinson señala la falta de indicaciones que ofrecen las distintas metodologías de enseñanza respecto a la primera lengua y aboga por un uso moderado y sistemático, especialmente en tareas de precisión (accuracy-oriented tasks). Atkinson, además, tiene muy claro que el papel de la Ll está intrínsecamente relacionado con el perfil del grupo: las clases monolingües tienen unas características especiales propias, y lo que en un entorno multilingüe puede resultar apropiado (el empleo exclusivo de la L2), quizá no lo sea para una clase que comparte la primera lengua (incluido en muchas ocasiones también el profesor). Por ello, su citado libro concluye con "10 tips for teachers who don't know their students' language" (Atkinson, 1993a: 105-106) a fin de sensibilizar a los profesores nativos de la L2 con respecto a la lengua y cultura de sus estudiantes. No obstante, su posición en esta cuestión se basa en su experiencia personal como profesor y no en los resultados de ninguna investigación.

Por su parte, Ernesto Macaro (2001) recoge en su artículo argumentos de distintos investigadores para no excluir la Ll del aula: su prohibición impediría establecer una conexión con los conceptos ya asentados en la primera lengua (Skinner); lo que cuenta no es la cantidad de input, sino la calidad (Dickson); el cambio de código es una destreza comunicativa fundamental (Hagen); excluir la primera lengua es una suerte de imperialismo lingüístico (Phillipson) y de exclusión de la cultura del aprendiz (Van der Walt; Prodromou, en Deller y Rinvolucri, 2002: 5), que genera actitudes negativas hacia la lengua meta (Stables and Wikeley), y que constituye, en definitiva, privar a los alumnos de una preeminente herramienta de aprendizaje. Su postura es que el uso de la Ll es muy recomendable especialmente en niveles más bajos, por motivos pedagógicos: "Banning codeswitching from classrooms, particularly beginner and lower-intermediate classrooms, may lead to a number of undesirable pedagogical practices, for example teacher domination of discourse or obstacles to learner-centered oral interaction" (Macaro, 2003: 42-43). Cole (1998) y Hito- 
tuzi (2006) ahondan en esta idea, pues reconocen que, en las clases de nivel inicial, la primera lengua puede utilizarse casi para cualquier acción realizada en el aula, tanto por parte del profesor como del alumno, desde la organización de la clase y de las tareas hasta las aclaraciones de comprensión pero, a la vez, insisten en que hay que animar al estudiante a emplear la lengua meta. Miles (2004: 6) añade que, si prescindimos de la primera lengua, estamos limitando el número de métodos y técnicas al alcance de los docentes de idiomas.

Por ello, muchos autores recomiendan, especialmente, el análisis contrastivo de L1 y L2 (Atkinson, 1987; Hopkins, 1988; Harbord, 1992; Butzkamm, 1998; Cole, 1998; Schweers, 1999; Sheen, 2000), a fin de aumentar la conciencia de los aprendices sobre las similitudes y diferencias de ambas lenguas, así como de los falsos amigos o los peligros de una traducción palabra por palabra. El uso comparativo y contrastivo de la Ll ayuda a profesores y aprendices a identificar las áreas más problemáticas del aprendizaje, y puede extenderse a los aspectos culturales igualmente. "Understanding linguistic differences between students' Ll and English may help the learners reduce interference from their first language", dice Chen (2006: 76). El ejemplo que nos muestra Helena Prins (2006) sobre la enseñanza de inglés en Taiwán lo ilustra claramente: las políticas de No-Mandarin-During-English-Time provocan graves errores de transferencia lingüística entre los alumnos, que podrían solucionarse introduciendo el análisis contrastivo en el aula y animando a los profesores a conocer la $\mathrm{Ll}$ de los alumnos a fin de comprender la fuente de algunos de sus errores. Aparte de las comparaciones gramaticales, también es posible establecer el análisis contrastivo a nivel semántico, pragmático y discursivo (Gabrielatos, 2001: 7).

El uso del Análisis contrastivo ha sido un recurso denostado largo tiempo por su inevitable asociación con el método de gramática-traducción y el audiolingüismo. Sin embargo, se trata de un proceso inevitable que los aprendices realizan consciente e inconscientemente, por lo que un empleo eficaz de esta técnica podría consistir en ayudarles no a traducir palabra por palabra o estructura por estructura, sino el significado social o funcional del mensaje completo (Weschler, 1997: 8). Chen (2006: 76) recomienda su uso en cursos específicos de destreza escrita, donde las interferencias con la primera lengua son evidentes, a juzgar por la amplia bibliografía que ha puesto de manifiesto este fenómeno. Él cita varios trabajos que analizan los errores de producción escrita de estudiantes de inglés, muchos de ellos achacables al recurso a la Ll. Por tanto, "A better understanding of the $\mathrm{Ll}$ influence in the process of EFL writing will help teachers know students' difficulties in learning English" (Chen, 2006). 
Por su parte, Castellotti y Moore (2002: 17) consideran que el recurso a la L1 únicamente es válido en la medida en que permite a los aprendices hallar las diferencias entre lenguas:

Mais si le retour à la matrice de la langue première apparaît comme la stratégie la plus fréquente pour la plupart des élèves, quels que soient leur âge et leur degré de contact avec la langue-cible, ce retour ne trouve une efficacité que dans la mesure où les élèves réussissent un mouvement de décentration qui leur permet de mettre en place des hypothèses de fonctionnement différencié.

Estos argumentos pueden encuadrarse en una línea cognitivista del aprendizaje de idiomas, basada en los procesos que operan en la mente del hablante no nativo. En esta misma tendencia podemos citar a O'Malley y Chamot (1990), cuyo Cognitive Academic Language Learning Approach (CALLA) reconoce en la primera lengua una herramienta de aprendizaje fundamental ${ }^{7}$, tal como había avanzado Corder (1981). “...The mother tongue represents a powerful resource that can be used in a number of ways to enhance learning..." (Gill, 2005: 1); "the students' L1 should be used as 'a tool for teaching English" (Hopkins, 1988: 19). También para Robert Lado (1957) se trataba de una herramienta esencial, pero no de aprendizaje, sino de enseñanza, porque el conocimiento de las diferencias y similitudes de L1 y L2 nos llevaría directamente a la naturaleza del aprendizaje. En esta línea podemos incluir el comentario de Robert Weschler (1997: 2): “... better to think of the inevitable influence of the L1 on the L2 as a potential aid or tool". Hoy en día, este empleo del análisis contrastivo es defendido por autoras tan respetadas como Patsy Lightbown y Nina Spada (1999), que recomiendan el contraste L1 - L2 en metodologías basadas en la atención a la forma (Doughty y Williams, 1998) puestas en práctica en clases monolingües.

Ferrer (2004: 6) considera estas comparaciones interlingüísticas como una herramienta más a disposición del profesor en sus explicaciones de corte gramatical, mientras que Prodromou (2001) resalta su valor como instrumento de activación de los conocimientos culturales para la adquisición de la L2. Algunos de los alumnos participantes en el estudio de Tang (2002) resaltaron

7 Prácticamente todos los autores que mencionamos emplean la palabra tool, "herramienta", para describir el papel de la Ll en el aula. Al igual que en clase se emplean dibujos, objetos reales y fotos para enseñar vocabulario a los alumnos, puesto que son herramientas a disposición del profesor, ¿por qué no se tiene esta misma visión de la primera lengua? ¿Por qué muchos docentes no consideran igual el empleo de diagramas, de imágenes u objetos y el uso de la L1? Es una de las preguntas que se hacen, entre otros, Paul Nation (2003: 5), y que da cuenta de la "especial" consideración que ha tenido la lengua materna en la enseñanza de idiomas. "The $\mathrm{Ll}$ needs to be seen as a useful tool that like other tools should be used where needed but should not be over-used" (Nation, 2003: 5). 
este uso también por parte de los aprendices, especialmente con los ejercicios de traducción. De hecho, en su estudio sobre el papel de la traducción en la enseñanza de idiomas, Zanier Visintin (2002) señala que traducir es una actividad automática que realizan los alumnos de todos los niveles, puesto que es así como funciona cognitivamente la asimilación de nueva información: construyéndola a partir del conocimiento previo. Buckmaster (2000: 2) va un paso más allá, al estimar que el uso de la L1 da poder (empowers) al aprendiz de L2 ${ }^{8}$ (siempre, en el contexto de clases monolingües). Afirmaciones similares son las vertidas por N. Eleni Pappamihiel, de la Universidad del Estado de Florida, en el foro de Stanley (2002): "I strongly believe use of the students' L1 TO SUPPORT L2 acquisition is appropriate", y cita como ejemplo la transferencia de habilidades cognitivas. Olivares (1995) menciona este uso aplicado a la destreza lectora en la clase de inglés, en la que propone emplear periódicos en L1 y L2. Llega incluso a vincular el progreso en la L2 con los conocimientos de la L1, que permitirán la transferencia de habilidades y estrategias: "With a strong base in L1, they will be able to transfer most of their knowledge, skills, and learning strategies to L2" (Olivares, 1995: 3). De hecho, muchas investigaciones sobre competencia lectora han tomado en consideración las habilidades equivalentes en Ll, analizando su impacto (Macaro, 2003: 128 y ss.), por no hablar de toda la tradición bibliográfica que relaciona la competencia general en L1 (por ejemplo, el grado de alfabetización y estudios) con la adquisición de una L2 (Auerbach, 1993).

Este papel facilitador de la L1 ha sido puesto de relieve por diversos autores, como Martín Martín (2001), Atkinson (1993a) o Tang (2002), a la vista de los resultados de su investigación: "The study also reveals that in the EFL classes observed Chinese plays only a supportive and facilitating role [...] the use of the mother tongue is only a means to the end of improving foreign language proficiency" (p. 4). El foro de discusión sobre la Ll editado por Karen Stanley (2002) nos ofrece algunos testimonios que ilustran estas afirmaciones, como el de Julia Reineman, cuyo profesor de español prohibía totalmente el uso de inglés tanto en clase como en su despacho, creándole en consecuencia enormes problemas de comprensión a la hora de aclarar dudas.

8 Frente a esta postura, también mantenida por Auerbach (1993), Polio (1994: 156) considera que "...it is possible to teach language to empower students using only the L2. [...] Empowering students is not so much an issue of what language is spoken but of what is done with the language and the extent to which students' ideas are encouraged and valued. [...] maintenance of the students' Lls, such maintenance does not guarantee empowerment either". 
En consecuencia, muchos autores (Antón y DiCamilla, 1999; Swain y Lapkin, 2000) ponen de manifiesto el papel de la primera lengua como herramienta cognitiva, que entra en juego especialmente en la realización de tareas de alta carga cognitiva. Por ejemplo, un estudio realizado por Cohen (1994, citado en Swain y Lapkin, 2000: 253) muestra cómo en un programa de inmersión en español, los alumnos que tenían que resolver problemas matemáticos cambiaban a su lengua materna, el inglés, para resolver las tareas más complicadas conceptualmente, es decir, para llevar a cabo los procesos mentales. Según Swain y Lapkin (2000), esta visión de la Ll como herramienta de procesamiento cognitivo contrasta fuertemente con la dominante durante décadas sobre la Ll como factor de transferencia. Por ello, prefieren la perspectiva neovygotskiana de la teoría sociocultural, según la cual el lenguaje se concibe como mecanismo de mediación en los procesos mentales que acompañan a las actividades sociales. Es la misma idea que subyace a las palabras de Wolfgang Butzkamm (2003: 31): "Monolingual lessons without the help of the mother tongue are extrinsically possible; however, monolingual learning is an intrinsic impossibility. No one can simply turn off what they already know".

En la bibliografía sobre tareas posee un lugar destacado la línea de estudios de tareas de colaboración para la resolución de problemas en el aula (Brooks y Donato, 1994; Antón y DiCamilla, 1999; De Guerrero y Villamil, 2000). En efecto, aunque algunos profesores lamentan que en las tareas realizadas en pequeños grupos los alumnos tiendan a agruparse en función de su primera lengua, lo cierto es que esta relación les permite apoyarse (the 'scaffolding' support) en la construcción de la L2: "... when a teacher uses the L1 in the TL classroom, the learner uses the L1 as a cognitive tool to help 'scaffold' his/her learning" (Turnbull y Arnett, 2002: 206). Algunos estudios que tratan las estrategias de aprendizaje (como el de Schmitt, 1997, citado por Cook, 2001a) muestran que el uso de la Ll es un recurso empleado por el 73\% de los alumnos que preguntan el significado de algo a sus compañeros. Aiva Drukovskis, profesora de inglés en New Jersey (Stanley, 2002), relata cómo en sus clases multilingües los alumnos tienden a agruparse en función de su lengua de origen, de modo que aquellos cuya primera lengua no es hablada por nadie más suelen abandonar las clases rápidamente, mientras que quienes han establecido "grupos de apoyo" en su Ll se quedan hasta el final del curso. Parece que su repercusión va más allá de lo lingüístico y se relaciona con factores afectivos (Crandall, en Stanley, 2002). En estos casos, la mayoría de investigadores resalta el valor de la Ll para la consecución de la tarea, especialmente en alumnos con niveles más bajos y en las tareas más complejas, pues el recurso a la lengua común sirve como herramienta de mediación, de andamiaje para los aprendices: 
For L2 learners, $\mathrm{L} 1$ as well as $\mathrm{L} 2$ can provide helpful mediation. Talk between peers who are collaborating in tasks is often in their common L1, which provides an efficient (and sometimes essential) medium for problem-solving and can enhance learning of both L2 and any academic subjects students are studying in the second language. (Saville-Troike, 2006: 113).

Desde el punto de vista de las teorías sociales del aprendizaje (Block, 2003; Lantolf y Thorne, 2006), el diálogo colaborativo es el medio esencial por el que el ser humano aprende. Específicamente en el aula de idiomas, el uso de la L1 ha sido estudiado por Antón y DiCamilla (1999), quienes hallan que, en este contexto, la Ll favorece la adquisición. Los investigadores encuentran tres funciones esenciales de la L1: "construction of scaffolded help, establishment of intersubjectivity, and use of private speech" (1999: 245). Es decir, andamiaje, intersubjetividad y habla privada. Es algo más que una herramienta metalingüística: es un medio para crear un espacio cognitivo y social en el que los alumnos se ayudan mutuamente a lo largo de toda la tarea. Esta visión es compartida por Brooks y Donato (1994: 268), para quienes el cambio de código es "a normal psycholinguistic process that facilitates L2 production and allow learners both to initiate and sustain verbal interaction with one another".

Uno de los trabajos más citados en este ámbito es el de Merrill Swain y Sharon Lapkin (2000), que pusieron de relieve el papel que desempeña la primera lengua en la realización de estas tareas de colaboración entre alumnos. $\mathrm{Su}$ estudio sugiere que negar este recurso a los aprendices les priva de una valiosísima herramienta cognitiva: "To insist that no use be made of the L1 in carrying out tasks that are both linguistically and cognitively complex is to deny the use of an important cognitive tool" (Swain y Lapkin, 2000: 268-269). La L1 sirve de apoyo al estudiante no solo en estos diálogos colaborativos para la realización de tareas, sino también a nivel individual, tal como demuestra el estudio de Lourdes Ortega (2005) sobre la fase de planning en el desarrollo de tareas. Entre las diversas estrategias mencionadas por los aprendices para completarlas, Ortega recoge el recurso a la L1, con frecuencia, en forma de traducción. También Elsa Auerbach (1993: 21) había resaltado las bondades de la Ll en la fase de planificación en las composiciones escritas en lengua meta, a partir del correspondiente estudio de Osburne y Harss-Covaleski (1991, en Auerbach, 1993: 21; también Wang y Wen, 2002).

En ese sentido, una de las afirmaciones más rotundas es la de Péter Medgyes (1992), quien sostiene que la principal característica que ha de poseer un profesor de inglés nativo es el conocimiento del idioma de sus alumnos. Afines a esta aseveración son las apreciaciones de Elsa Auerbach (1993), David Atkinson (1993a) en su libro Teaching monolingual classes, Simon Cole (1998), 
Peter Dash (2002) o las de David Barker (2003) sobre la enseñanza de inglés en Japón. Él establece claramente la distinción entre utilizar la Ll y conocer la Ll del alumnado 9 . Parece existir una línea de conexión entre no emplear la Ll en el aula y no tener conocimientos sobre ella, aunque Barker recuerda las ventajas de conocer la lengua del país donde uno enseña la suya como nativo, independientemente de su empleo en clase. Si de lo que se trata es de aprender idiomas, el profesor tendría que predicar con el ejemplo: "Using Ll in the classroom shows my students I've learnt at least some of their language and shows respect. It also shows that I am a learner and can make mistakes", dice Buckmaster (2000: 2). Para Elsa Auerbach (1993: 25), durante mucho tiempo se ha dado por sentado que el profesor de ESL no ha de conocer la lengua de sus alumnos, sino que le basta el conocimiento del inglés y de las teorías, métodos, enfoques e investigación lingüística. Bien al contrario, Tomasz Pilch, profesor de inglés en Polonia, reconoce que saber ambas gramáticas permite al docente presentar algunas estructuras de la lengua meta más rápidamente y trabajar en los posibles errores derivados de las similitudes o divergencias (Stanley, 2002). "Personally speaking I would hate to learn a language with a teacher who didn't speak my own language, especially at a lower level", afirma Buckmaster (2000: 1), adoptando el papel de alumno. También Costas Gabrielatos (2001: 8) es muy claro al respecto:

Teachers need to be knowledgeable about the similarities and differences between the learners' native language and the target language at the semantic, morphosyntactic, pragmatic and discourse level, as well as be aware of the opportunities and pitfalls of Ll use in ELT and proceed according to principled frameworks.

Incluso Cole (1998), en su artículo sobre la enseñanza comunicativa de inglés en Japón, presenta una tabla de pequeñas frases en japonés para el profesor que no posea conocimientos de japonés pero quiera emplear un mínimo de Ll en el aula. Estas ideas están en consonancia con la opinión de algunos investigadores como Wilga M. Rivers $(1972,1981)$, que defiende que el uso de la Ll del alumno acelera el proceso de aprendizaje de la lengua meta (también Pilch, en Stanley, 2002). Además, no olvidemos que, en el caso de las lenguas extranjeras, su enseñanza corre muchas veces a cargo de profesores no nativos de esas lenguas y sí de la Ll de los aprendices, lo cual haría aún más absurda una docencia exclusivamente en lengua meta (Miles, 2004: 9). Peter Dash (2002) muestra ampliamente un buen ejemplo de estas situaciones, al describir la situación de la enseñanza actual del inglés en Corea, en donde no

9 Distinción que no hallamos, por ejemplo, en Hopkins (1988) o Larrea (2002), que mezclan ambos. 
han resultado efectivas las políticas de L2-only, puesto que los diversos informes y estudios realizados por las instituciones y el gobierno revelan que tanto alumnos como profesores prefieren un enfoque bilingüe.

La contrapartida la ofrecen testimonios como el del mismo Dash, y también Zacharias (2003: 81), en cuyo estudio algunos profesores empleaban la L1 porque sus conocimientos en L2 (inglés, en este caso) no eran lo suficientemente sólidos como para conducir la clase entera en lengua meta.

Desde otro punto de vista, W. E. Rutherford (1987) señala el papel que desempeñan los conocimientos previos del alumno que operan en la adquisición de una segunda lengua, argumentando que, sin ellos, "no language learning would be possible at all" (1987: 8; también Hopkins, 1988: 19). Hitotuzi reconoce igualmente el valor de esta herramienta: "...it seems learner $\mathrm{Ll}$ is a tool that neither the teacher nor the learner can afford to dispense with" (2006: 169), pero hace una llamada a la precaución en su uso, para que no resulte contraproducente (también Gabrielatos, 2001: 6). De la misma opinión es Prins (2006: 4), que considera que los profesores de idiomas deben partir de las experiencias lingüísticas y de aprendizaje previas de los aprendices y trabajar a partir de ellas, haciendo un uso inteligente de la primera lengua. Al fin y al cabo, "there is no evidence that the use of the first language in the classroom constrains the learning of the second language". Mario Rinvolucri (2003), uno de los grandes defensores del empleo de la primera lengua en el aula, considera natural emplear la primera lengua al aprender la segunda, al igual que cuando aprendemos cualquier otra cosa, siempre partimos de lo que ya sabemos, de lo que nos es familiar. Y en el caso de la segunda lengua, la referencia más cercana es nuestra primera lengua. Los grandes investigadores en estrategias de aprendizaje, como Ellen Bialystok (1990), siempre han reconocido el valor de los conocimientos previos a la hora de incorporar información nueva, y es frecuente entre los docentes la creencia, sustentada por diversas investigaciones, de que los niños que conocen mejor su primera lengua obtienen mejores resultados en la segunda (Cook, 2001b; Spada y Lightbown, 2002).

Todas estas experiencias previas forman parte de lo que se conoce como multicompetencia (Cook, 1991; 1999), concepto que hace referencia a la competencia comunicativa de los hablantes bilingües y multilingües, cuya capacidad lingüística no equivale a la superposición de varios hablantes monolingües, sino que conforma unas habilidades y competencias únicas: “...knowledge of two or more languages results in a unique and complex competence that is not equal to the sum of knowledge of monolingual speakers of those languages" (Kecskes y Papp, 2000: xxiv). Esta perspectiva multilingüe contrasta fuertemente con el mono- 
lingüismo aparentemente reinante en la Lingüística aplicada durante décadas (e, incluso, en amplias parcelas de la Lingüística general, como es el caso de las investigaciones de Chomsky, 1965). Esta visión concebía el aprendizaje de lenguas como la adquisición de una competencia monolingüe en L2, comparando insistentemente al aprendiz con el modelo nativo, al que indefectiblemente había de aspirar. Sin embargo, desde los años noventa existe una corriente de estudios que aboga por dar un paso hacia adelante desde la visión monolingüe y centrada en la sintaxis del hablante hacia una perspectiva multilingüe, holística, más centrada en el significado y la comunicación. "The key idea is that the person who speaks more than one language should be considered in their own right, not as a monolingual who has tacked another language onto their repertoire" (Cook, 2001b: 195). Desde esta perspectiva, la referencia ya no es el hablante nativo, sino tanto el aprendiz como el usuario de segundas lenguas (L2 users and L2 learners, Cook, 1999), no solo en lo que se refiere a la enseñanza de idiomas, sino también para la Lingüística aplicada en general ${ }^{10}$.

Esta nueva línea de investigación ha llevado a los investigadores de ASL a analizar el dinamismo del desarrollo multilingüe, deteniéndose en cuestiones como la influencia de la $\mathrm{L} 2$ en la $\mathrm{L}^{11}{ }^{11}$, la transferencia de normas y patrones de interacción sociocultural de una lengua a otra, el procesamiento del lenguaje y del pensamiento, la selección léxica, la transferencia de destrezas, el impacto de la distancia interlingüística, los conocimientos metalingüísticos y pragmáticos de L1 y L2 que implica la multicompetencia, su evaluación y la concepción del aula como un espacio multilingüe per se. Todo ello comporta, desde luego, un acercamiento multidisciplinar. El libro de Kecskes y Papp Foreign Language and Mother Tongue (2000) es una buena muestra de ello, aplicado al estudio de la influencia de la L2 en el desarrollo lingüístico de la L1.

10 En ese sentido, el artículo de Cook (1999) resulta muy clarificador, pues ofrece una profunda descripción del concepto de hablante nativo frente al usuario de una L2 y revisa el privilegiado estatus del que el primero ha gozado en los estudios de ASL. Continúa con una caracterización de los hablantes multicompetentes frente a los monolingües y concluye con las implicaciones que todo ello tiene para la enseñanza de idiomas. Su reivindicación es constante: "L2 users are not monolingual native speakers and never will be [...] L2 users have to be looked at in their own right as genuine L2 users, not as imitation native speakers" (Cook, 1999: 195).

11 "English children who learn Italian for an hour a week learn to read more rapidly in English", comenta Cook (2001b: 196) a modo de ejemplo de la influencia de una L2 en el uso de la primera lengua. Conviene recordar, no obstante, que existe además la posibilidad de una influencia negativa, es decir, que la fluidez y corrección en la Ll se vean mermadas porque el hablante esté inmerso en el aprendizaje de la L2. Odlin (2005) también se refiere a la posible influencia interlingüística de una L3 en la L2. 
Cabe citar aquí también un interesantísimo proyecto llevado a cabo en Canadá por The Ottawa Carleton District Shool Board (OCDSB), que consiste en proporcionar a los alumnos más desaventajados en la clase de inglés como segunda lengua instrucción en su primera lengua, de modo que los resultados de la $\mathrm{L} 1$ repercutan en un progreso en la $\mathrm{L} 2$ :

The less formal schooling a student has received in the first language, the less likely they are to be successful as they move into the upper grades. [...] Providing first language instructional support to students with limited or no first language schooling in their home country may make a significant difference in their academic achievement [in the L2] (Thibault, 2001).

Este ejemplo es buena muestra de las implicaciones del concepto de multicompetencia, al ilustrar claramente la idea de que los multilingües no consisten en varios hablantes monolingües superpuestos ${ }^{12}$, sino que sus habilidades implican los conocimientos lingüísticos globales, no compartimentados. Así lo entiende también el MCER (Consejo de Europa, 2001: 4 y ss.) cuando se refiere al plurilingüismo y a las competencias plurilingüe y pluricultural (p. 131 y ss.; p. 167 y ss.). Esta visión tiene inmediatas consecuencias en la moderna didáctica de lenguas, tal como Castellotti y Moore (2002: 20) apuntan, en el marco de sus trabajos para el Consejo de Europa:

Des enseignements ainsi axés sur une optimisation didactique de la proximité inter-linguistique doivent en particulier permettre de valoriser l'expertise des apprenants en leur donnant l'occasion de se servir de leurs compétences linguistiques, et de mettre à l'épreuve leurs intuitions sur le langage et les moyens de transférer leurs compétences d'un contexte à un autre. L'objectif est la mise en valeur et la construction de compétences transversales, qui peuvent être réactivées quelles que soient les langues apprises. Dans ces nouvelles perspectives, les rôles d'aide à la communication et à l'apprentissage de la langue première, ou d'autres langues médiatrices (comme l'anglais), devraient pouvoir trouver leur place dans la réflexion pédagogique des enseignants.

Este punto de vista comporta también una revisión del concepto de hablante nativo y de la proficiencia que un no nativo alcanza. Por mucho que algunos aprendices muestren una competencia comunicativa similar a la de los nativos, sus conocimientos lingüísticos (por ejemplo, la intuición gramatical) siempre serán distintos. Pero la perspectiva de la multicompetencia no ve al aprendiz de lenguas como un hablante deficiente, sino multicompetente, y ello obliga a los estudios de Adquisición de segundas lenguas a replantearse el papel del uso de la L1 y de otras lenguas en el aula de idiomas (Edstrom, 2006: 277).

12 En este sentido, recomiendo especialmente la lectura del texto "Non-native Speakers Exist!”, de Zoltán Dörnyei, publicado en García Laborda, 2006: 3-4. En él, Dörnyei reflexiona sobre su condición de hablante no nativo de inglés y sus distintas competencias lingüísticas y comunicativas en L1 y L2, comparándolas. 
Desde esta óptica, cabe afirmar que la Ll está siempre presente en la mente de los aprendices de idiomas (Wang y Wen, 2002; Gibson y Hufeisen, 2003; Choong, 2006), por lo que también forma parte de esos conocimientos previos: "...one must consider that the learner has intricate knowledge of an $\mathrm{Ll}$ and that this knowledge necessarily interfaces with his or her developing knowledge of the L2" (Belz, 2002: 211). Resultaría artificial entonces pretender que el alumno es una tabula rasa para evitar este uso: "Inclusion of the $\mathrm{Ll}$ in classrooms also helps legitimatize the L2 learner's multicompetent mind rather than artificially compartimentalizing the two or more languages" (Choong, 2006: 3; Zhou, 2003). Sin citar expresamente la palabra multicompetencia, Weschler (1997: 5) resalta el papel fundamental que desempeña la primera lengua en el procesamiento del significado de la L2 y, a su vez, el rol central que ocupa la cuestión del significado en la comunicación.

Más cauto resulta Vivian Cook (2001a), cuya pretensión no es tanto fomentar el uso de la Ll en el aula cuanto eliminar todo rastro de arrepentimiento y rechazo entre quienes la emplean. Considera que la visión de la L1 como una técnica al alcance del profesor nos descubre nuevos modos para su empleo en clase: desde la expresión del significado y la explicación gramatical hasta la organización de la clase, en el caso de profesores; como elemento de un aprendizaje colaborativo y una estrategia individual de uso para el alumno. "The first language can be a useful element in creating authentic L2 users rather than something to be shunned at all costs" (Cook, 2001a: 1).

Cook se plantea este uso de la Ll en términos de eficacia (¿qué tareas son más efectivas a través de la Ll?), aprendizaje (¿la Ll nos ayuda en el aprendizaje de una L2?), naturalidad (¿los participantes se sienten más cómodos al tratar algunos temas o funciones en L1 en lugar de en L2, según muestran los estudios de cambio de código?) y relevancia externa (¿el uso de ambas lenguas ayuda a los alumnos a dominar usos específicos de la L2?), con la contrapartida de que cualquier uso de la primera lengua en el aula supone restar tiempo de empleo de la lengua meta.

En muchos casos, investigadores y docentes reconocen que la lengua meta ha de ser la principal lengua en el aula, tanto lengua de instrucción como de comunicación, aunque un uso ocasional de la primera lengua resulta beneficioso (Atkinson, 1993a), por ejemplo, para que los alumnos no sientan que se les imponen la lengua y la cultura metas (Auerbach, 1993; Larrea, 2002). Algunos testimonios que ilustran esta postura son los siguientes: “... The prudent use of L2 in the English classroom affirms the value of our students' L1 as their primary means of communication and cultural expression"; "... bringing Spanish into the English classes has made learning English appear to be less of a 
threat to their vernacular" (Schweers, 1999: 3); "Teachers need to show respect for the learners' $\mathrm{Ll}$ and need to avoid doing things that make the $\mathrm{Ll}$ seem inferior to English" (Nation, 2003: 7). A este respecto, uno de los ensayos más claros es el capítulo de Julie A. Belz "Identity, Deficiency, and First Language Use in Foreign Language Education" (2002), incluido en el libro de Carl Blyth The Sociolinguistics of Foreign Language Classrooms: Contributions of the Native, Near-Native, and Non-Native Speaker. En él, Belz reivindica el papel de la L1 por su vinculación con la identidad de los aprendices, partiendo de la base de que el aprendizaje de una lengua extranjera moldea la identidad lingüística y social de los estudiantes:

I assume that the use of a particular linguistic code (language, dialect or register) can represent a particular identity; thus, the use of more than one language may function as the representation of multiple speaker identities [...] The denial of the use of a certain language could be interpreted as a truncation of one's linguistic identity and therefore represent a form of symbolic violence to the self. (Belz, 2002: 209-210).

En relación con la identidad del aprendiz de idiomas hallamos el concepto de inversión (investment), propuesto por Norton (1995) para explicar la decisión y el esfuerzo de aprender una lengua desde el punto de vista de lo que dicho aprendizaje supone para la identidad del hablante. "If learners invest in a second language, they do so with the understanding that they will acquire a wider range of symbolic and material resources, which will in turn increase the value of their cultural capital" (Norton, 1995: 17). Por tanto, el empleo de la $\mathrm{Ll}$ puede responder a la negativa de invertir en una segunda lengua por sus repercusiones en la consideración social del aprendiz, sea cual fuere.

Para otros autores, como Zacharias (2003: 34), el empleo de la Ll tiene más que ver con la situación real fuera del aula, en la que el multilingüismo es la tónica: "... in a context like Indonesia, where most people are bilinguals, banning the mother tongue creates an artificially constructed environment in the classroom, which disregards the bilingual reality that surrounds it. The use of the mother tongue is one indication that the class is communicative and "real".

Otra de las aplicaciones de la L1 más citadas en los estudios es la representación de conceptos abstractos, ya que la traducción a la Ll suele ser una técnica muy socorrida: "... abstract concepts simply cannot be conveyed through obvious gestures, pictures and commands", subraya Weschler (1997: 4); también Zacharias (2003: 35), para quien "translation is a 'lubricant' that makes the learning and teaching process of the target language go faster and smoother". Los resultados del trabajo de Tang (2002: 3) apuntan ese mismo uso por parte de los docentes de inglés en China y es igualmente señalado como de gran utilidad 
entre los alumnos. "Try miming 'although", decía Mario Rinvolucri en un congreso de TESOL en España hace algunos años. Lo mismo comenta Bill Snyder, de Turquía, cuando trataba de explicar qué significa "sin embargo". La única técnica que funcionó para que los alumnos comprendieran el concepto fue la traducción a la L1 (Stanley, 2002). "Sometimes a quick translated word makes life easier" (Tibbetts, en Stanely, 2002).

De otro lado, algunos autores relacionan su empleo con factores afectivos; por ejemplo, para crear una atmósfera relajada (Zacharias, 2003: 81), y como modo de hacer bajar el filtro afectivo de los aprendices para, en consecuencia, reducir su ansiedad (Duff y Polio, 1990: 163; Cole, 1998; Wang, 2002: 5; Levine, 2003: 346; Miles, 2004: 9; Rolin-Ianziti y Varshney, 2008). De hecho, la hipótesis de la que parte Levine en su investigación es que cuanto mayor es el uso de la lengua meta, más crece la ansiedad de los aprendices, hipótesis que su estudio refuta, y que ya hemos comentado. Sea como fuere, su conclusión es clara: "...denying a role to the Ll would appear to be a futile endeavor" (Levine, 2003: 355). Atkinson (1993a: 13) recomienda el uso de la Ll especialmente en clases monolingües de niveles bajos para evitar la frustración y el estrés que sienten los aprendices: "One hundred-per-cent direct method can be especially stressful and frustrating -limited use of the Ll can have a powerful, positive effect here [...] occasional use of the Ll gives them the opportunity to show that they are intelligent, sophisticated people"13. De la misma opinión son los profesores escoceses entrevistados por R. Mitchell (1988), que perciben la L2 como un factor de estrés.

También menciona esta vinculación con los factores afectivos Sandra Fotos (2001: 329-330), cuyo estudio presenta una visión de corte sociolingüístico respecto al papel de la Ll dentro de las comunidades de habla, haciendo especial hincapié en las ventajas del cambio de código:

...foreign language classrooms can become linguistic microclimates which function as distinct speech communities whithin the native culture. Use of the students' Ll during study of their second language (L2) within this microclimate can enhance community membership, creating favourable affective conditions for learning, and can also serve as a strategy for increasing the salience of input from the target language. In particular, language alternation within the same utterance, or codeswitching, can serve a dual function: promoting SLA through negotiation of meaning and focus on form, and fostering the students' sense of their bilingual identity.

13 Frente a este argumento, que relaciona los usos de la primera lengua con factores afectivos, Turnbull y Arnett (2002: 206) consideran el punto de vista opuesto, en relación con la motivación: un uso excesivo de la Ll puede provocar un desinterés en el alumnado, puesto que nada hay más motivador en el aula de idiomas que constatar los propios progresos en la L2, a través de un uso máximo de ésta en el aula. 
De acuerdo con Fotos (2001: 331), una visión positiva del papel de la L1 implica la concepción del aula de idiomas como una comunidad cultural, en consonancia con los estudios que la describen como un microcosmos, un microclima lingüístico, especialmente en el ámbito de los trabajos sobre comunicación intercultural. Es una visión similar a la que mantienen los estudios de construcción colaborativa del significado, inspirados en la tradición sociocultural de Vygotsky ya mencionada, y para quienes el aula de lenguas se convierte en una comunidad de práctica (también en Cook, 2001b: 157).

Otra perspectiva sociolingüística es la ofrecida por Julie Belz (2002), que aboga por un uso consciente de los cambios de código en el aula de lenguas, ofreciendo pautas de uso a los alumnos y reconociendo sus virtudes en las clases multilingües.

Como vemos, la mayoría de los expertos que trata el tema lo hace poniendo de relieve los beneficios del uso de la Ll. Incluso Auerbach (1993: 18) menciona que el uso de la Ll es capaz de atraer alumnos a las clases de ESL. Bien al contrario, Robert Weschler (1997: 1) se sirve de la estrategia opuesta, al rebatir las políticas de uso exclusivo de la L2:

Where did we get this idea that "only English" should be spoken in the English classroom? Is it based on any cohesive theory or substantiated research? Or more likely, is it the result of blind acceptance of certain dogma which conveniently serves the best interests of native speaker teachers? It is my purpose to show that, while our students are here to learn English (the L2), there are many possible means toward that end, that there is a time and a place for everything, and that one of those means is, without doubt, the timely use of the students' first language (the L1).

Más adelante, reitera: "The assumption that the English-only, direct method can be applied equally well to any size and type of class and any level or content of language is simply false" (Weschler, 1997: 4); "There is no reason why a teacher shouldn't take advantage of the students' shared knowledge in bridging the gap to what they don't yet know" (Weschler, 1997: 5). Para él, la conclusión es clara: "...the question is not if, but rather to what degree, students 'think' in the L1 when trying to comprehend and express themselves in the L2" (Weschler, 1997: 12).

Casi todos los argumentos expuestos hasta ahora han sido recogidos por Wolfgang Butzkamm (2003: 31 y ss.) en una suerte de decálogo que reúne diez máximas sobre el uso de la lengua materna, y que reproducimos a continuación:

- Maxim 1: The FL learners must build upon existing skills and knowledge acquired in and through the MT. 
- Maxim 2: Ersatz-techniques ${ }^{14}$ for meaning-conveyance function less well than the MT and can even be harmful.

- Maxim 3: MT aids make it easier to conduct whole lessons in the foreign language. Pupils gain in confidence and, paradoxically, become less dependent on their MT.

- Maxim 4: MT aids can promote authentic, message-orientated communication than might be found in lessons where they are avoided.

- Maxim 5: MT techniques allow teachers to use richer, more authentic texts sooner. This means more comprehensible input and faster acquisition.

- Maxim 6: Bilingual techniques allow teachers to bypass the grammatical progression of textbooks. No postponement of the subjunctive.

- Maxim 7: We need to associate the new with the old. To exclude MT links would deprive us of the richest source for building cross-linguistic networks. No quarantine for MT cognates and related words.

- Maxim 8: It is not possible to avoid interference, but it can be greatly reduced.

- Maxim 9: Paradoxically, the counter-productive haphazard use of the mother tongue may be an unwanted side-effect of the doctrine of monolingualism.

- Maxim 10: All newly-acquired FL items have to sink roots in our minds which are eventually deep enough for the items to function independently of the MT.

Podemos concluir, pues, con las palabras finales de Simon Gill (2005: 4) en su artículo sobre los usos de la Ll en el aula:

I do feel strongly that the L1 should not be used simply as an optional 'spice' [...]. It's much more basic than that; it is something that every learner in every classroom possesses, and I believe that we, as teachers, need to be clear in our minds, not about whether it should be used (by us, by learners, or by both), because, whether we like it or not, it will be anyway, but about, first, when and why it should be used, and then, once we've got that straight, how.

Esta gran variedad de argumentos que acabamos de presentar, así como el peso del razonamiento expuesto por Simon Gill, constituyen una profunda revisión del papel de la primera lengua en el aula y dan cuenta de la verdadera dimensión y alcance de la influencia de la L1 en la adquisición de una lengua adicional, desaconsejando, en consecuencia, cualquier postura radical en cuanto a su exclusión de la clase de idiomas.

14 Se refiere a explicaciones reformulatorias o definitorias para explicar el significado de una palabra, en lugar de proporcionar directamente la traducción. 


\section{Conclusión}

A lo largo de estas páginas hemos analizado en detalle la multidimensionalidad del papel que desempeña la lengua nativa de los aprendices durante la adquisición de una lengua extranjera. Han sido y son muchas las razones aludidas para rechazar su inclusión en el aula de idiomas, relacionadas sobre todo con la cantidad de exposición al input de la lengua meta necesaria para que se produzca la asimilación de la L2. Ahora bien, también son numerosos los investigadores que han puesto de relieve la intrincada relación existente entre L1 y L2 en la mente de los estudiantes, que el docente de idiomas de ninguna manera puede ignorar.

En ese sentido, y con respecto al debate en torno al uso de la Ll en el aula de L2, cabe citar los tres planteamientos teóricos que propone Ernesto Macaro (2001: 535) a la hora de tratar esta cuestión:

1. La postura virtual (The Virtual Position): la clase es como el país meta, por lo que el objetivo ha de ser la total exclusión de la primera lengua. No existe ninguna razón didáctica para emplearla, por lo que es posible prescindir de ella siempre que el profesor posea las habilidades necesarias para tal fin.

2. La postura máxima (The Maximal Position): no hay razón pedagógica para utilizar la Ll. Sin embargo, no existen las condiciones perfectas de enseñanza y aprendizaje y, por tanto, los profesores han de recurrir a ella.

3. La postura óptima (The Optimal Position): hay cierto valor didáctico en el uso de la L1, puesto que sabemos que puede mejorar algunos aspectos del aprendizaje. Por ello, debe haber una constante exploración de los principios pedagógicos que regulen cuándo y cómo se justifica su empleo en el aula de idiomas.

Según Macaro, la bibliografía especializada parece sugerir que la postura virtual es insostenible, y que la segunda de ellas suele conducir a sentimientos de culpabilidad y desconfianza entre los docentes. Este profesor de la Universidad de Oxford parece decantarse implícitamente por la tercera opción.

Sea como fuere, a la vista de todos los pros y contras que hemos discutido en estas páginas, Weschler (1997: 12) propone que el profesor comience su primer día de clase diciendo: "Class. We are here to learn English. Let's do whatever we need to do". Y eso implica, exactamente, plantearse sin rodeos la pregunta que da título a nuestro artículo respecto al uso de la Ll en el aula de L2: ¿por qué no? 


\section{Referencias bibliográficas}

Antón, Marta y Frederick J. DiCamilla (1999): "Socio-Cognitive Functions of L1 Collaborative Interaction in the L2 Classroom”, The Modern Language Journal, 83 (2), págs. $233-247$.

Atkinson, David (1987): "The mother tongue in the classroom: a neglected resource?", ELT Journal, 41 (4), págs. 241 - 247.

Atkinson, David (1993a): Teaching monolingual classes. Londres, Longman.

Atkinson, David (1995): "English Only in the Classroom: why do we do it?, The Polish Teacher Trainer, 3 (1). http://ettc.uwb.edu.pl/strony/ptt/feb95/8.html

Auerbach, Elsa Roberts (1993): "Reexamining English Only in the ESL Classroom", TESOL Quarterly, 27 (1), págs. 9 - 32.

Auerbach, Elsa Roberts (1994): "The author responds..." [respuesta al comentario de Charlene Polio a su artículo de 1993], TESOL Quarterly, 28, págs. 157 - 161.

Barker, David (2003): "Why English Teachers in Japan need to learn Japanese", The Language Teacher Online. http://www.jalt-publications.org/tlt/articles/2003/02/barker

Belz, Julie A. (2002): "Identity, Deficiency, and First Language Use in Foreign Language Education", en BLYTH, Carl (ed.), págs. 209 - 248.

http://eric.ed.gov/ERICDocs/data/ericdocs2sql/content_storage_01/0000019b/80/1b/7f/ bb.pdf

Bialystok, Ellen (1990): Communication strategies. A psychological analysis of second language use. Oxford, Basil Blackwell.

Blyth, Carl S. (2003): The Sociolinguistics of Foreign Language Classrooms: Contributions of the Native, Near-Native, and Non-Native Speaker. AAUSC Issues in Language Program Direction. Boston, Heinle.

Block, David (2003): The Social Turn in Second Language Acquisition. Edimburgo, Edinburgh University Press.

Brooks, Frank B. y Richard Donato (1994): "Vygotskyan approaches to understanding foreign language learner discourse during communicative tasks", Hispania, 77 (2), págs. $262-274$.

Buckmaster, Robert (2000): "Using L1: What Kind of Sin?", IATEFL Poland Newsletter, 18. http://www.iatefl.org.pl/nletter/nletter18/nlet18_2.html

Burden, Peter (2000): "The use of the students' mother tongue in monolingual English 'conversation' classes at Japanese universities”, The Language Teacher Online Editor, June 2000. http://www.jalt-publications.org/tlt/articles/2000/06/burden

Butzkamm, Wolfgang (1998): "Code-Switching in a Bilingual History Lesson: The Mother Tongue as a Conversational Lubricant", International Journal of Bilingual Education and Bilingualism, 1 (2), págs. 81 - 99.

Butzkamm, Wolfgang (2003): "We only learn language once. The role of the mother tongue in FL classrooms: death of a dogma", Language Learning Journal, Winter, 28, págs. $29-39$.

Carless, David (2008): "Student use of the mother tongue in the task-based classroom", ELT Journal, 62 (4), págs. 331 - 338. 
Castellotti, Véronique (1997a): "Langue étrangère et français en milieu scolaire: didactiser l'alternance?", en Castellotti, Véronique y Danièle Moore (eds), págs. 401 - 410.

Castellotti, Véronique y Danièle Moore (2002): REPRESENTATIONS SOCIALES DES LANGUES ET ENSEIGNEMENTS. Guide pour l'élaboration des politiques linguistiques éducatives en Europe - De la diversité linguistique à l'éducation plurilingue. Division des politiques linguistiques, Conseil de l'Europe. www.coe.int/t/dg4/ linguistic/Source/CastellottiMooreFR.pdf

Centeno-Cortés, Beatriz y Antonio F. Jiménez Jiménez (2004): "Problem-solving tasks in a foreign language: The importance of the $\mathrm{Ll}$ in private verbal thinking", International Journal of Applied Linguistics, 14 (1), págs. 7 - 35.

Chambers, Francine (1991): "Promoting use of the target language in the classroom", Language Learning Journal, 4, págs. 27 - 31. www.ittmfl.org.uk/modules/teaching/lc/paperlc2.PDF

Chaudron, Craig (1988): Second language classrooms: Research on teaching and learning. Cambridge, Cambridge University Press.

Chen, Li-Ling (2006): "The Effect of the Use of Ll in a Multimedia Tutorial on Grammar Learning: An Error Analysis of Taiwanese Beginning EFL Learners' English Essays", Asian EFL Journal, 8 (2). http://www.asian-efl-journal.com/June_06_llc.php

Chomsky, Noam (1965): Aspects of the Theory of Syntax. Cambridge, Mass., MIT Press.

Choong, K. Philip (2006): "Multicompetence and Second Language Teaching", Teachers College, Columbia University Working Papers in TESOL \& Applied Linguistics, 6 (1). The Forum. http://www.tc.columbia.edu/academic/tesol/WJFiles/pdf/Choong.pdf

Cohen, E. G. (1994): "Reestructuring the classroom: conditions for productive small groups", Review of Educational Research, 64. 1 - 35.

Cole, Simon (1998): "The Use of L1 in Communicative English Classrooms", The Language Teacher Online 22.12. http://www.jalt-publications.org/tlt/files/98/dec/cole.html.

Consejo de Europa (2001): Common European Framework of Reference for Languages: Learning, Teaching, Assessment. Cambridge, CUP. Versión española disponible en el Centro Virtual Cervantes. http://213.4.108.140/obref/marco/default.htm

Cook, Vivian (1991), "The poverty-of-the-stimulus argument and multi-competence", Second Language Research, 7, 2, págs. $103-117$.

Cook, Vivian (1999): "Going Beyond the Native Speaker in Language Teaching", TESOL Quarterly, 33 (2), págs. 185 - 209.

Cook, Vivian (2001a): "Using the First Language in the Classroom", Canadian Modern Language Review, 57 (3), págs. 402 - 423.

Cook, Vivian (2001b): Second Language Learning and Language Teaching. Oxford University Press.

Corder, Stephen Pit (1981): Error analysis and interlanguage. Oxford, Oxford University Press.

Corcoran, James (2009): "Rethinking Ll Use in the ESL/EFL Classroom", Contact. Teachers of English as a Second Language of Ontario, 35 (1), págs. 8 - 13. 
Cunningham Florez, MaryAnn (2000): "Native Language in the Beginning Adult ESL Classroom: To Use or Not To Use", Eric documents, págs. 3 - 6.

http://eric.ed.gov/ERICWebPortal/custom/portlets/recordDetails/detailmini.jsp?_nfpb=t rue\&_\&ERICExtSearch_SearchValue_0=ED442312\&ERICExtSearch_SearchType _0=no\&accno=ED442312

Çelik, Servet (2008): "Opening the door: an examination of mother tongue use in foreign language classrooms”, Hacettepe Üniversitesi Journal of Education, 34, págs. $75-85$.

Dash, Peter S. (2002): "English Only (EO) In The Classroom: Time For a Reality Check?", Asian EFL Journal, 4 (4). http://www.asian-efl-journal.com/dec_02_pd.pdf

Deller, Sheelagh y Mario Rinvolucri (2002): Using the Mother Tongue: Making the Most of the Learner's Language. First Person Publishing.

De Guerrero, Maria y Olga S. Villamil (2000): "Activating the ZPD: Mutual scaffolding in L2 peer revision", The Modern Language Journal, 84 (1), págs. 51 - 68.

Doughty, Catherine y Jessica Williams (eds.) (1998): Focus on form in classroom second language acquisition. Cambridge, Cambridge University Press.

Duff, Patricia A. (2005): "Heteroglossia in Foreign Language Classrooms: Research, Debates, and Issues". Public lecture at Monash University (Australia). http://www.arts.monash.edu/lcl/research/seminars/duff-handout.pdf

Duff, Patricia A. y Charlene G. Polio (1990): "How Much Foreign Language is There in the Foreign Language Classroom?", The Modern Language Journal, 74 (2), págs. $154-166$.

Edstrom, Anne (2006): "L1 Use in the L2 Classroom: One Teacher's Self-Evaluation", The Canadian Modern Language Review, 63 (2), págs. 275 - 292.

Ellis, Rod (1984): Classroom Second Language Development. Oxford, Pergamon.

Ellis, Rod (1985): Understanding Second Language Acquisition. Oxford, Oxford University Press.

Ferrer, Vincent (2004): "The mother tongue in the classroom: cross-linguistic comparisons, noticing and explicit knowledge", Teaching English Worldwide.

http://www.teachenglishworldwide.com/Articles/Ferrer_mother\%20tongue\%20in $\% 20$ the\%20classroom.pdf

Fotos, Sandra (2001): “Codeswitching by Japan's Unrecognized Bilinguals: Japanese University Students' Use of Their Native Language as a Learning Strategy", en Goebel Noguchi, Mary y Sandra Fotos (eds.): Studies in Japanese Bilingualism. Multilingual Matters, págs. 329 - 352.

Franklin, Carole E. M. (1990): "Teaching in the target language: problems and prospects", Language Learning Journal, 2, págs. 20 - 24.

Gabrielatos, Costas (2001): "Ll Use in ELT: Not a Skeleton, but a Bone of Contention. A response to Prodromou". TESOL Greece Newsletter, 70. 6-9. También en Bridges, 6 May. 33 - 35. http://www.tesolgreece.com/nl/70/7001.html

Galindo Merino, Ma Mar (2005a): "La incorporación del nivel pragmático a la investigación sobre los procesos de transferencia en la adquisición de segundas lenguas", Estudios de Lingüística de la Universidad de Alicante, 19, págs. 137 - 155. 
Galindo Merino, Ma Mar (2005b): "La transferencia pragmática en el aprendizaje de E/LE”, en Álvarez, A. et alii (2006): La competencia pragmática y la enseñanza del español como lengua extranjera. Actas del XVI Congreso Internacional de ASELE. Oviedo, Universidad de Oviedo, págs. 289 - 297. http://cvc.cervantes.es/ensenanza/biblioteca_ele/asele/pdf/16/16_0287.pdf

Galindo Merino, Ma Mar (2009): "Los métodos de enseñanza de idiomas desde la perspectiva del aprendiz y su lengua materna”, Interlingüística, nº 19, págs. 546 - 560 . http://www.joveneslinguistas.org/interlinguistica/docs/Interlinguistica_2008.pdf

García Laborda, Jesús (ed.) (2006): "Forum: Native Or Non-Native - Can We Still Wonder Who Is Better?", TESL-EJ, Teaching English as a Second or Foreign Language, 10 (1). http://tesl-ej.org/ej37/f1.pdf

Gibson, Martha y Britta Hufeisen (2003): "Investigating the Role of Prior Foreign Language Knowledge: Translating from an Unknown into a Known Foreign Language", en Cenoz, Jasone, Hufeisen, Britta y Ulrike Jessner (eds.) (2003): The Multilingual Lexicon. Kluwer Academic Publishers, Holanda, págs. 87 - 102.

Gill, Simon (2005): "The L1 in the L2 Classroom", Humanising Language Teaching, Year 7, Issue 5. http://www.hltmag.co.uk/sep05/mart03.htm

González García, Diego (2009): "Uso de la Ll en el aula de ELE: beneficio o perjuicio", E-Excellence, Biblioteca de recursos electrónicos de Humanidades, Madrid, Liceus. www.liceus.com.

Harbord, John (1992): "The use of the mother tongue in the classroom", ELT Journal, 46 (4), Oxford University Press, págs. 350 - 355.

Hitotuzi, Nilton (2006): "The Learner's Mother Tongue in the L2 Learning-Teaching Symbiosis (La lengua materna del estudiante en la simbiosis entre enseñanza y aprendizaje de una segunda lengua)", PROFILE, 7, Jan./dec. 2006, págs. 160 - 172. http://www.scielo.org.co/scielo.php?script=sci_arttext\&pid=S1657$07902006000100012 \& \operatorname{lng}=\mathrm{en} \& \mathrm{nrm}=\mathrm{iso}$ http://www.scielo.org. co/pdf/prf/n7/n7a12.pdf

Hopkins, Sheona M. (1988): "Use of mother tongue in the teaching of English as a second language to adults", Multi-Mag, págs. $18-24$.

Kecskes, Istvan y Tünde Papp (2000): Foreign Language and Mother Tongue. Mahwah, New Jersey, Lawrence Erlbaum Associates.

Kim, Sun Hee Ok y Catherine Elder (2005): "Language choices and pedagogic functions in the foreign language classroom: a cross-linguistic functional analysis of teacher talk", Language Teaching Research, 9 (4), págs. 355 - 380.

Krashen, Stephen (1980): "The input hypothesis", en Alatis, J. (ed.) (1980): Current issues in bilingual education. Washington, D.C., Georgetown University Press, págs. $168-180$.

Krashen, Stephen (1981): Second language acquisition and second language learning. Oxford, Pergamon.

Krashen, Stephen (1985): The input Hypothesis: Issues and Implications. Londres, Longman. 
Lado, Robert (1957): Linguistics across Cultures: Applied linguistics for language teachers. Ann Arbor, University of Michigan Press.

Lantolf, James P. y Steven L. Thorne (2006): Sociocultural theory and the genesis of L2 development. Oxford, Oxford University Press.

Larrea, Edgar (2002): "Should we (or should we not) use L1 in the communicative English classroom?". APPROACH. A Journal for English Language Teaching in Cuba, December.

http://www.up.edu.pe/idiomas/programas_ingles/go_beyond_nuevo.php?pid=7

Lavan, Cindy (2001): "Help! They're Using Too Much English! The Problem of L1 vs. L2 in the Immersion Classroom", ACIE Newsletter, 4 (2).

http://www.carla.umn.edu/immersion/acie/vol4/Feb2001.pdf

Levine, Glenn S. (2003): "Student and Instructor Beliefs and Attitudes about Target Language Use, First Language Use, and Anxiety: Report of a Questionnaire Study", The Modern Language Journal, 87 (3), págs. 343 - 364.

Lightbown, Patsy M. y Nina Spada (1999): How languages are learned. Oxford, Oxford University Press, $2006^{3}$.

Lynch, Tony (1996): Communication in the Language Classroom. Oxford University Press.

Macaro, Ernesto (2001): "Analysing Student Teachers' Codeswitching in Foreign Language Classrooms: Theories and Decision Making”, The Modern Language Journal, 85 (4), págs. $531-548$.

Macaro, Ernesto (2003): Teaching and Learning a Second Language. A Guide to Recent Research and its Applications. Londres, Continuum.

Martín Martín, José Miguel (2000a): La lengua materna en el aprendizaje de una segunda lengua. Sevilla, Universidad de Sevilla.

Martín Martín, José Miguel (2000b): "El español en el aula de inglés. Un estudio empírico", ELIA. Estudios de Lingüística Inglesa Aplicada, 1. Universidad de Sevilla, págs. $81-91$.

http://www.institucional.us.es/revistas/revistas/elia/pdf/1/6-Jose\%20Miguel.pdf

Martín Martín, José Miguel (2001): "Nuevas tendencias en el uso de la Ll", ELIA. Estudios de Lingüística Inglesa Aplicada, 2, págs. 159 - 169.

http://www.institucional.us.es/revistas/revistas/elia/pdf/2/12.Jose\%20miguel.pdf

Martín Martín, José Miguel (2004): "Sobre lo contrastivo y el conocimiento lingüístico previo en la enseñanza del español”, en Ruhstaller, Stefan y Francisco Lorenzo Berguillos (coords.): La competencia lingüística y comunicativa en el aprendizaje del español como lengua extranjera. Universidad Pablo de Olavide, Sevilla, y Madrid, Edinumen, págs. $11-23$.

Medgyes, Péter (1992): “Native or non-native: who's worth more?, English Language Teaching Journal, 46 (4), págs. 340 - 349.

Miles, Richard (2004): Evaluating the use of L1 in the English language classroom. Memoria de máster para la School of Humanities de la Universidad de Birmingham, UK. http://www.cels.bham.ac.uk/resources/essays/Milesdiss.pdf 
Miquel, Lourdes y Neus Sans (eds.) (1993): Didáctica del español como lengua extranjera E/LE. Madrid, Fundación Actilibre, Col. Expolingua, vol. 1 (1995, vol. 2; 1996, vol. 3; 1999, vol. 4; 2002, vol. 5).

Mitchell, Rosamond (1988): Communicative Language Teaching in Practice. Londres, CILT.

Nation, Paul (2003): "The role of the first language in foreign language learning", Asian EFL Journal, 5 (2). http://asian-efl-journal.com/june_2003_pn.pdf

Norton, Bonny (1995): "Social identity, investment, and language learning", TESOL Quarterly, 29, págs. 9 - 31.

Nussbaum, Luci (1991): "La lengua materna en clase de lengua extranjera: entre la ayuda y el obstáculo", Signos. Teoría y Práctica de la educación, 4, Julio-Diciembre, págs. $36-47$.

Odlin, Terence (2005): "Crosslinguistic influence and conceptual transfer: what are the concepts?", Annual Review of Applied Linguistics, 25, págs. 3 - 25.

Olivares, Rafael A. (1995): "Using the Newspaper in the Bilingual Classroom", The Journal of Educational Issue of Language Minority Students, 15. Boise State University. http://www.ncela.gwu.edu/pubs/jeilms/vol15/usingthenews.htm

O’Malley, J. Michael y Anna Uhl Chamot (1990): Language learning strategies. Nueva York, Cambridge University Press.

Ortega, Lourdes (2005): "What do learners plan? Learner-driven attention to form during pre-task planning", en Ellis, Rod (ed.): Planning and Task Performance in a Second Language. Amsterdam / Philadelphia, John Benjamins, págs. 77 - 110.

Phillipson, Robert (1992): Linguistic imperialism. Oxford, Oxford University Press.

Polio, Charlene (1994): "Comments on Elsa Roberts Auerbach's "Reexamining English Only in the ESL Classroom", TESOL Quarterly, 28, págs. 153 - 157.

Prins, Helena (2006): "Conquering Chinese English in the ESL Classroom". The Internet TESL Journal, Vol. XII, $\mathrm{n}^{\circ}$ 11. http://iteslj.org/Techniques/PrinsChinglish.html

Prodromou, Luke (2001): "From Mother Tongue to Other Tongue", TESOL Greece, Newsletter 67 y Bridges, 5. http://web.archive.org/web/20021119093709/www.thracenet.gr/bridges/bridges5/From+Mother+Tongue+to+Other+Tonge.html

Rinvolucri, Mario (2003): "Contrasting language exercises using mother tongue sensibly”, BBC/British Council Teaching English. IATEFL 2003. http://www.teachingenglish.org.uk/iatefl2003/magali_26.shtml

Rivers, Wilga M. (1972): Speaking in many tongues: Essays in foreign-language teaching. Chicago, Chicago University Press.

Rivers, Wilga M. (1981): Teaching foreign-language skills. Chicago, Chicago University Press.

Rodríguez Juárez, Carolina y Gina Oxbrow (2008): "L1 in the EFL classroom: more a help than a hindrance?", Porta Linguarum, 9, págs. 93 - 109. http://www.ugr.es/ portalin/articulos/PL_numero9/7\%20Carolina.pdf http://dialnet.unirioja.es/servlet/articulo? codigo=2530654 
Rolin-Ianziti, Jeanne y Rachel Varshney (2008): "Students' Views Regarding the Use of the First Language: An Exploratory Study in a Tertiary Context Maximizing Target Language Use", Canadian Modern Language Review, 65 (2), págs. 249 - 273.

Rutherford, William E. (1987): Second language grammar. Learning and teaching. Nueva York, Longman.

Salaberri Ramiro, Ma Sagrario (1993): Uso del inglés en el aula. Oxford, Heinemann.

Saville-Troike, Muriel (2006): Introducing Second Language Acquisition. CUP, Cambridge Introductions to Language and Linguistics.

Schweers, C. William Jr. (1999): "Using L1 in the L2 Classroom", English Teaching Forum Online, 37 (2). http://draft.eca.state.gov/forum/vols/vol37/no2/p6.htm

Sheen, Ron (2000): "A response to Spada and Lightbown: 'Instruction, first language influence, and developmental readiness in second language acquisition'", The Modern Language Journal, 84, págs. 102 - 106.

Spada, Nina y Patsy M. Lightbown (2002): "L1 and L2 in the education of Inuit children in northern Quebec: abilities and perceptions", Language and Education, 16 (3), págs. $212-240$.

Stanley, Karen (ed.) (2002): "Using The First Language In Second Language Instruction: If, When, Why and How Much?. (TESL-EJ Forum)". TESL-EJ. Teaching English as a Second or Foreign Language, 5 (4). http://tesl-ej.org/ej20/fl.html

Storch, Neomy y Gillian Wigglesworth (2003): "Is there a role for the use of the L1 in an L2 setting?", TESOL Quarterly, 37 (4), págs. 760 - 770.

Straub, Jennifer (2006): "How do I increase student motivation to speak French in an L2 classroom/French immersion setting?", The Ontario Action Researcher, Archive, 5.41E. http://www.nipissingu.ca/oar/archive-Vol5Nol-V514E.htm

Swain, Merrill (1985): "Communicative competence: some roles of comprehensible input and comprehensible output in its development", en Gass, S. y C. Madden (eds.): Input in second language acquisition. Rowley, Mass., Newbury House, págs. 235 - 253.

Swain, Merrill y Sharon Lapkin (2000): "Task-based second language learning: The uses of the first language", Language Teaching Research, 4 (3), págs. $253-274$.

Tang, Jinlan (2002): "Using L1 in the English Classroom”, English Teaching Forum Online. Bureau of Educational and Cultural Affairs, 40 (1). http://exchanges.state.gov/forum/ vols/vol40/nol/p36.pdf

Tarone, Elaine y Merrill Swain (1995): "A sociolinguistic perspective on second language use in immersion classrooms", The Modern Language Journal, 79, págs. 166 - 178.

Thibault, Nicole (2001): "Using L1 to Support L2 Learning: Best Practices the OCDSB Way", CALST/ACPLS (Canadian Association of Second Language Teachers). http://www.caslt.org/research/12_11.htm

Thoms, Joshua, Liao, Jianling y Anja Szustak (2005): "The Use of L1 in an L2 On-Line Chat Activity”, The Canadian Modern Language Review, 62 (1), págs. 161 - 182.

Turnbull, Miles y Katy Arnett (2002): "Teachers' uses of the target and first languages in second and foreign language classrooms", Annual Review of Applied Linguistics, 22, págs. $204-218$. 
Turnbull, Miles y Jennifer Dailey-O'Cain (eds.) (2009): First Language Use in Second and Foreign Language Learning. Multilingual Matters.

Wajnryb, Ruth (2004): "Using the Mother Tongue. Review of Deller \& Rinvolucri's 2002 book", English Australia Journal, 21 (2), págs. 88 - 89.

http://www.englishaustralia.com.au/index.cgi?E=hsvalidator\&template=journal\&e file $=$ admin $\& X=S \&$ Lev $1=5 \&$ Lev $2=3$

Wang, Catherine (2002): "First Language or Second Language?", Essay Depot. http://www.essaydepto.com/essayme/1882/index.php

Wang, Wenyu y Quifang Wen (2002): "L1 use in the L2 composing process: An exploratory study of 16 Chinese EFL writers", Journal of Second Language Writing, 11 (3), págs. $225-246$.

Weschler, Robert (1997): "Uses of Japanese (L1) in the English Classroom: Introducing the Functional-Translation Method", The Internet TESL Journal, III, 11. http://iteslj. org/Articles/Weschler-UsingLl.html

Zacharias, Nugrahenny T. (2003): A survey of tertiary teachers' beliefs about English Language Teaching in Indonesia with regard to the role of English as a global language, MA-ELT Thesis, Institute for English Language Education, Assumption University of Thailand, Asian EFL Journal, June 2007. http://www.asian-efl-journal.com/thesis_N_Zacharias.pdf

Zanier Visintin, Alessio (2002): "Un posible papel de la traducción en la enseñanza de idiomas", División de Estudios Internacionales y Humanidades, Universidad de Quintana Roo (México).

http://dzibanche.biblos.uqroo.mx/cursos_linea2/azanier/art_un_posible_papel_de _la_traducc.htm

Zhou, Jin (2003): "New wine in an old bottle: innovative EFL classrooms in China", IATEFL Issues 172. http://www.iatefl.org/content/newsletter/172.php 